\title{
COVID-19 Vaccination in Pregnancy, Paediatrics, Immunocompromised Patients, and Persons with History of Allergy or Prior SARS-CoV-2 Infection: Overview of Current Recommendations and Pre- and Post-Marketing Evidence for Vaccine Efficacy and Safety
}

\author{
Nicoletta Luxi ${ }^{1}$ - Alexia Giovanazzi ${ }^{1} \cdot$ Annalisa Capuano $^{2}$ - Salvatore Crisafulli ${ }^{3}$ Paola Maria Cutroneo ${ }^{4}$. \\ Maria Pia Fantini ${ }^{5}$. Carmen Ferrajolo ${ }^{2}$. Ugo Moretti ${ }^{1}$ - Elisabetta Poluzzi ${ }^{6}$. Emanuel Raschi ${ }^{6}$. Claudia Ravaldi $^{7}$. \\ Chiara Reno $^{5}$ - Marco Tuccori ${ }^{8}$ - Alfredo Vannacci ${ }^{7}$ - Giovanna Zanoni ${ }^{9} \cdot$ Gianluca Trifirò ${ }^{1}$ (1) - Ilmiovaccino COVID19 \\ collaborating group
}

Accepted: 21 October 2021 / Published online: 5 November 2021

(c) The Author(s), under exclusive licence to Springer Nature Switzerland AG 2021

\begin{abstract}
To date, four vaccines have been authorised for emergency use and under conditional approval by the European Medicines Agency to prevent COVID-19: Comirnaty, COVID-19 Vaccine Janssen, Spikevax (previously COVID-19 Vaccine Moderna) and Vaxzevria (previously COVID-19 Vaccine AstraZeneca). Although the benefit-risk profile of these vaccines was proven to be largely favourable in the general population, evidence in special cohorts initially excluded from the pivotal trials, such as pregnant and breastfeeding women, children/adolescents, immunocompromised people and persons with a history of allergy or previous SARS-CoV-2 infection, is still limited. In this narrative review, we critically overview pre- and post-marketing evidence on the potential benefits and risks of marketed COVID-19 vaccines in the above-mentioned special cohorts. In addition, we summarise the recommendations of the scientific societies and regulatory agencies about COVID-19 primary prevention in the same vaccinee categories.
\end{abstract}

\section{Introduction}

To date, four COVID-19 vaccines have been authorised for emergency use in Europe starting from December 2020: the COVID-19 m-RNA vaccines Comirnaty (developed by Pfizer/Biontech) and Spikevax (previously COVID-19 Vaccine Moderna) and the COVID-19 viral vector vaccines COVID-19 Vaccine Janssen and Vaxzevria (previously COVID-19 Vaccine AstraZeneca). This has happened incredibly fast as a result of a collective effort by regulatory agencies, pharmaceutical companies and the scientific

Nicoletta Luxi and Alexia Giovanazzi contributed equally to the paper as first authors.

The members of Ilmiovaccino COVID19 collaborating group are listed in acknowledgements section.

Gianluca Trifirò

gianluca.trifiro@univr.it

Extended author information available on the last page of the article

\section{Key Points}

Evidence on the benefit-risk profile of COVID-19 vaccines in special cohorts, such as pregnant and breastfeeding women, children/adolescents, immunocompromised people, and persons with a history of allergy or previous SARS-CoV-2 infection, is still limited.

Due to the higher risk of SARS-CoV-2 infection and severe COVID-19, vaccination is currently recommended in these special cohorts. COVID-19 vaccination for children and adolescents is still debated.

Ongoing large-scale studies will provide clinically relevant data in the frailest populations to better inform the worldwide COVID-19 vaccination campaign. 
community. However, all the COVID-19 vaccines have been approved on the basis of a so-called conditional approval. For this reason, vaccine effectiveness and safety will be closely monitored in the post-marketing real-world setting, and vaccine manufacturers have been requested to provide more comprehensive data for additional re-evaluation of the vaccine benefit-risk profile by regulatory agencies.

Overall, COVID-19 vaccines have been shown to be highly effective and safe in the general population [1-4]. However, data on efficacy and safety may vary across different categories, based on the underlying risk of developing SARS-CoV-2 infection and severe COVID-19, as well as the likelihood of experiencing adverse reactions following immunisation. The aim of this narrative review is to critically summarise pre- and post-marketing evidence on potential benefits and risks of marketed COVID-19 vaccines in special cohorts that have not been included in initial pivotal clinical trials, such as pregnant and breastfeeding women, children/adolescents, immunocompromised people, and persons with a history of allergy or previous SARS-CoV-2 infection. In addition, an overview of scientific societies' and regulatory agencies' recommendations about COVID-19 vaccines in those categories is provided.

\section{Evidence of Benefits and Risks of COVID-19 Vaccines in Special Cohorts}

For each cohort under study, we explored information on the pre-marketing evidence of COVID-19 vaccine benefit-risk profile by searching ongoing studies registered in ClinicalTrials.gov until July 22, 2021 (Table 1) as well revising pivotal clinical trials. In addition, MEDLINE and Google Scholar databases were searched to identify observational studies, case series and case reports concerning effectiveness and risks associated with COVID-19 vaccines in special cohorts from the post-marketing setting. Finally, websites of the most important international/national scientific societies and regulatory agencies have been explored to identify recommendations on COVID-19 vaccination in the abovementioned categories of people. We searched terms related to COVID-19 vaccines, pregnant/lactating women, children/ adolescents, immunocompromised, allergy and SARSCoV-2 infection.

\subsection{Children and Adolescents}

\subsubsection{Epidemiology of SARS-CoV-2 Infection}

The initial studies conducted during the first wave of the COVID-19 pandemic suggested that children and adolescents could be less susceptible to SARS-CoV-2 infection compared with adults [5]. In Vo' (Veneto, Italy), nasopharyngeal swabs carried out during two separate surveys on $85.9 \%$ and $71.5 \%$ of the population, respectively, did not identify SARS-CoV-2-infected cases among the 234 tested children aged $0-10$ years, including those living with infected family members, in line with other studies conducted at that time [6-8]. The measured cumulative incidence per 1000 children aged 0-9 years in three Italian regions initially hard hit by the pandemic (Lombardy, Emilia-Romagna and Veneto) was lower compared with the rest of the population [9]. Preliminary results of a seroprevalence study of IgG against SARS-CoV-2 in Italy, carried out between May 25, 2020, and July 15, 2020, on a sample of 64,660 people, highlighted that the lowest value was found in the age class $0-5$ years (1.3\%) [10]. Moreover, early reports showed that only a small proportion of COVID19 cases were among children and adolescents (aged 0-18 years), with an incidence that increased with rising age: as of July 2020, of all cases of COVID-19 reported in the European Union/European Economic Area (EU/EEA) and United Kingdom (UK), only $4 \%$ were children/adolescents, of which $24 \%$ were in those under 5 years of age, $32 \%$ between 5 and 11 years and $44 \%$ between 12 and 18 years $[11,12]$. These figures have been the basis of different vaccination policies scaled up, starting from the end of 2020; for example, in the UK, vaccination is offered to all healthy adolescents aged $>16$ years, and children aged $>12$ years if they have an underlying condition that makes them high risk for severe COVID-19 [13]. However, a more recent study on primary and secondary school attendees emphasises that there could be no difference in terms of infection incidence among age groups, even if evidence is far from being definitive [14]. This observation could be explained considering that, especially during the 'first wave' of the pandemic from February to May 2020, SARS-CoV-2-positive children could have been underrepresented as they are more likely to have asymptomatic or mild infection, with better overall outcomes than adults. The most frequent symptoms in children are cough, fever and fatigue, but also atypical symptoms such as vomiting and diarrhoea are common; anosmia and ageusia are mainly referred to in pre-adolescents and adolescents, as younger children are often unable to describe these kinds of symptoms [15-17]. Children and adolescents are also much less likely to be hospitalised or have fatal outcomes [15, 17]. As of July 17, 2021, the cumulative rates of laboratoryconfirmed COVID-19-associated hospitalisations in the US were 62.8 per 100,000 in the age class $0-4$ years, 21.3 in the age class 5-11 years and 59.2 in the age class $12-17$ years, while in the population aged 18 years and older, the cumulative rate was 732.2 per 100,000 [18].

Furthermore, pre-existing medical conditions have been suggested as a risk factor for severe disease and intensive care unit (ICU) admission in children and adolescents [19]. As compared with children and adolescents, infants and 


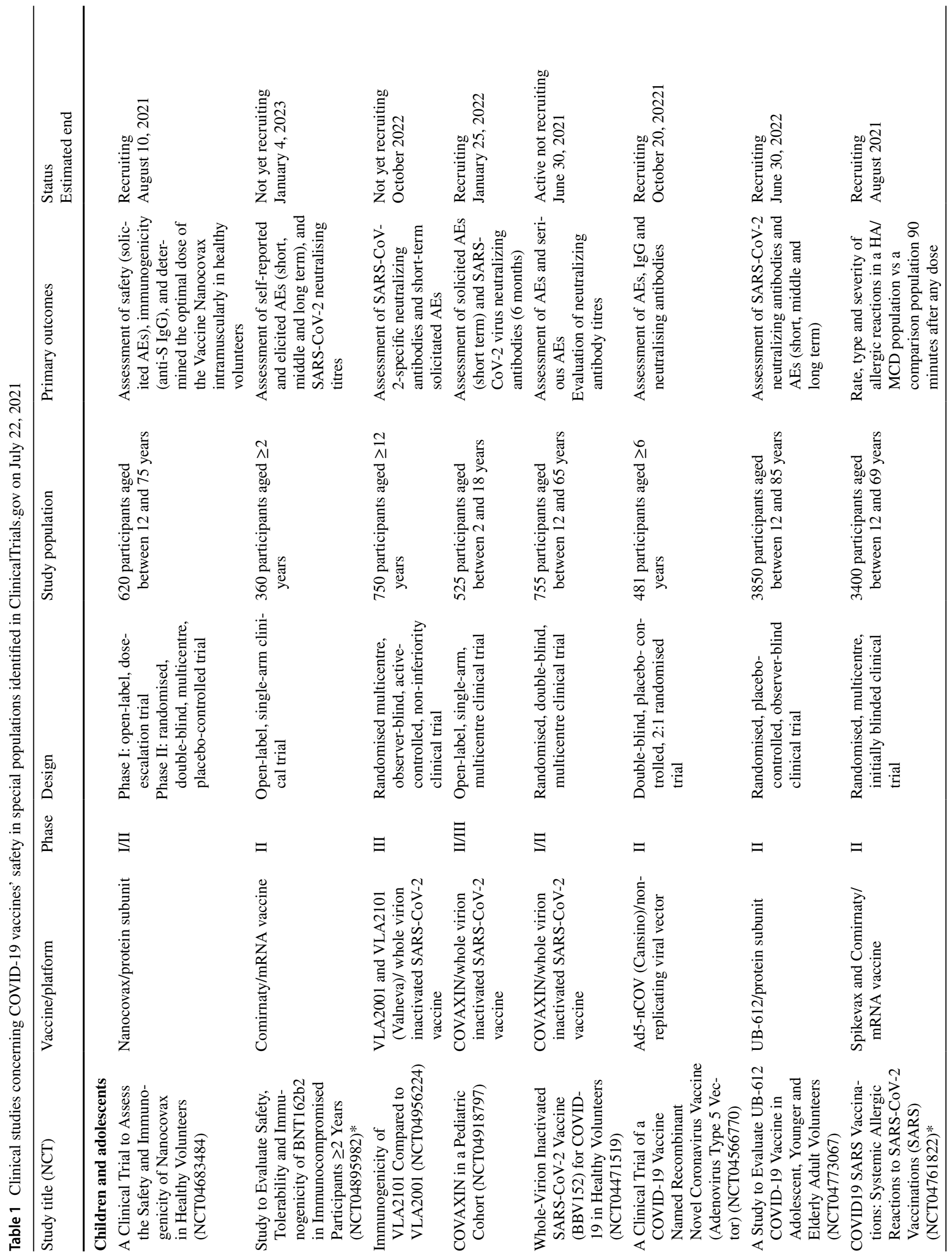




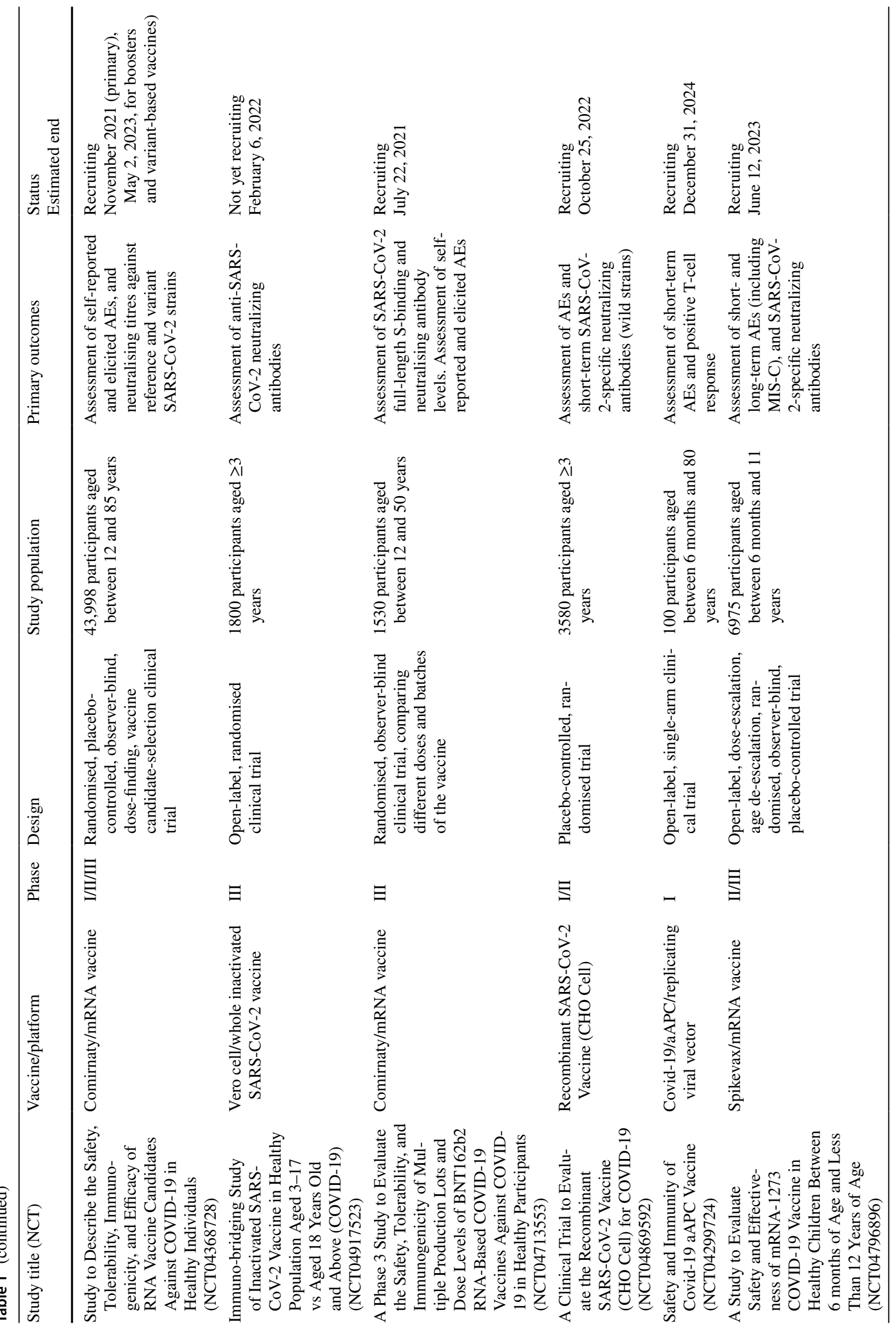




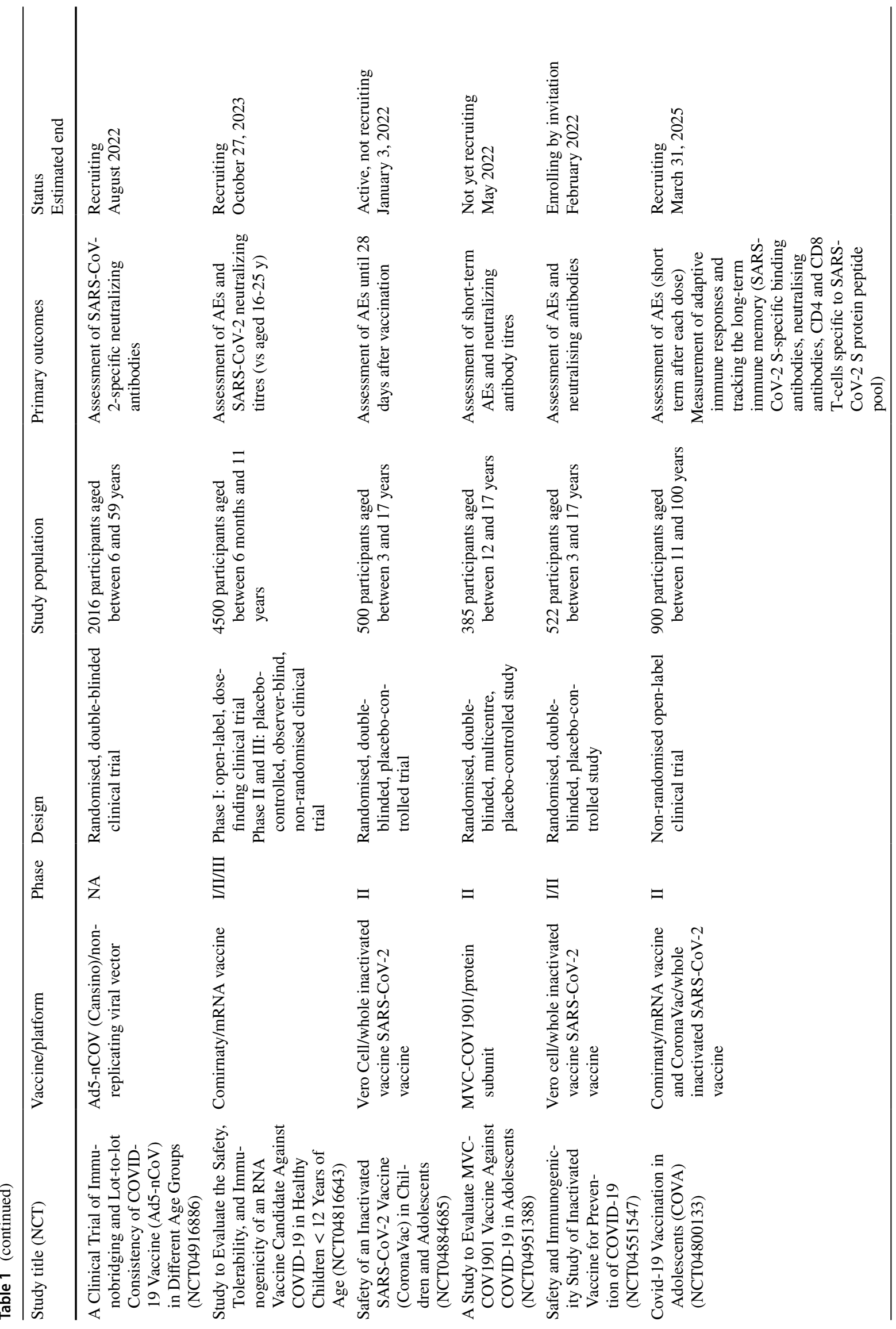




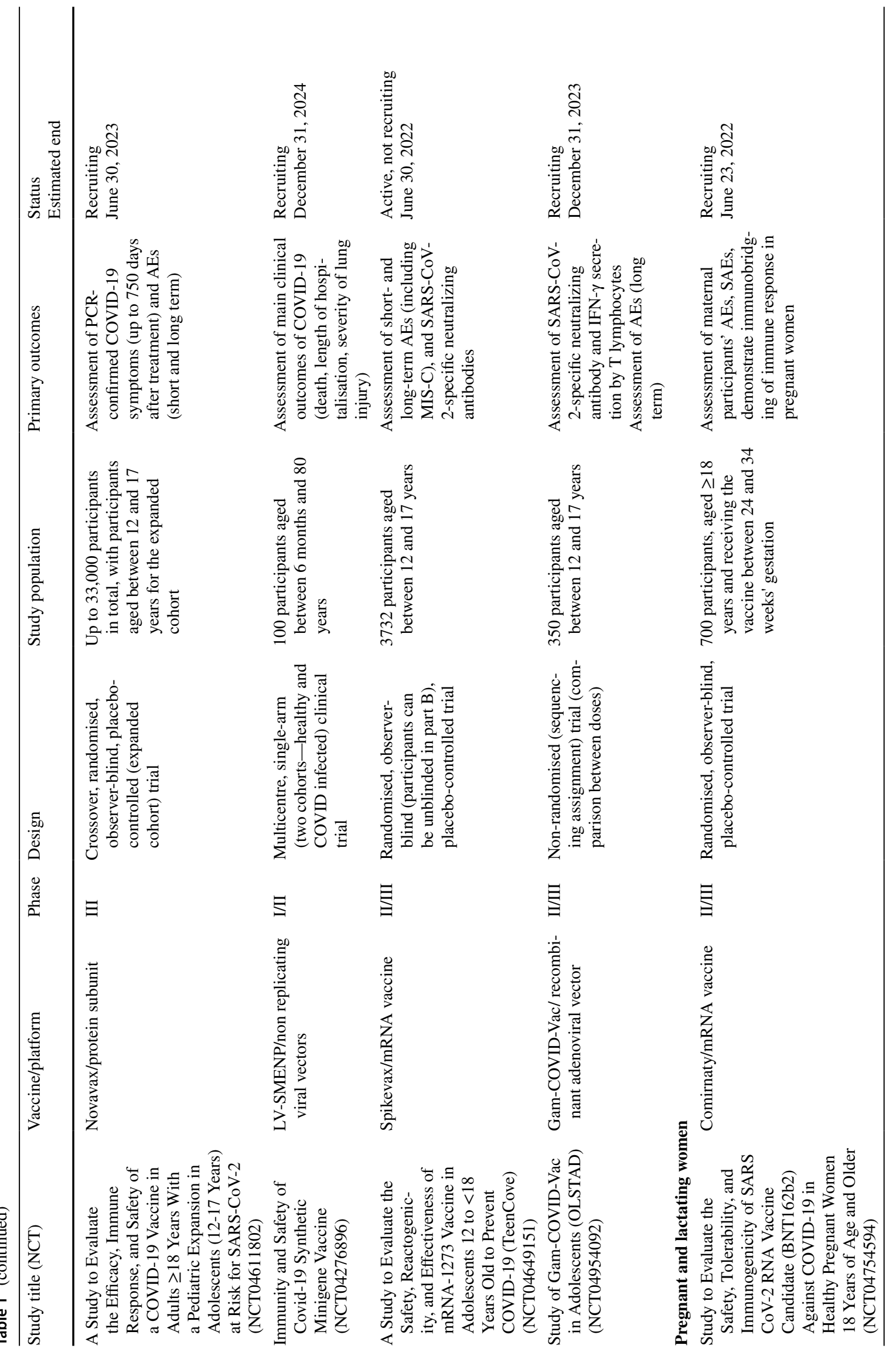




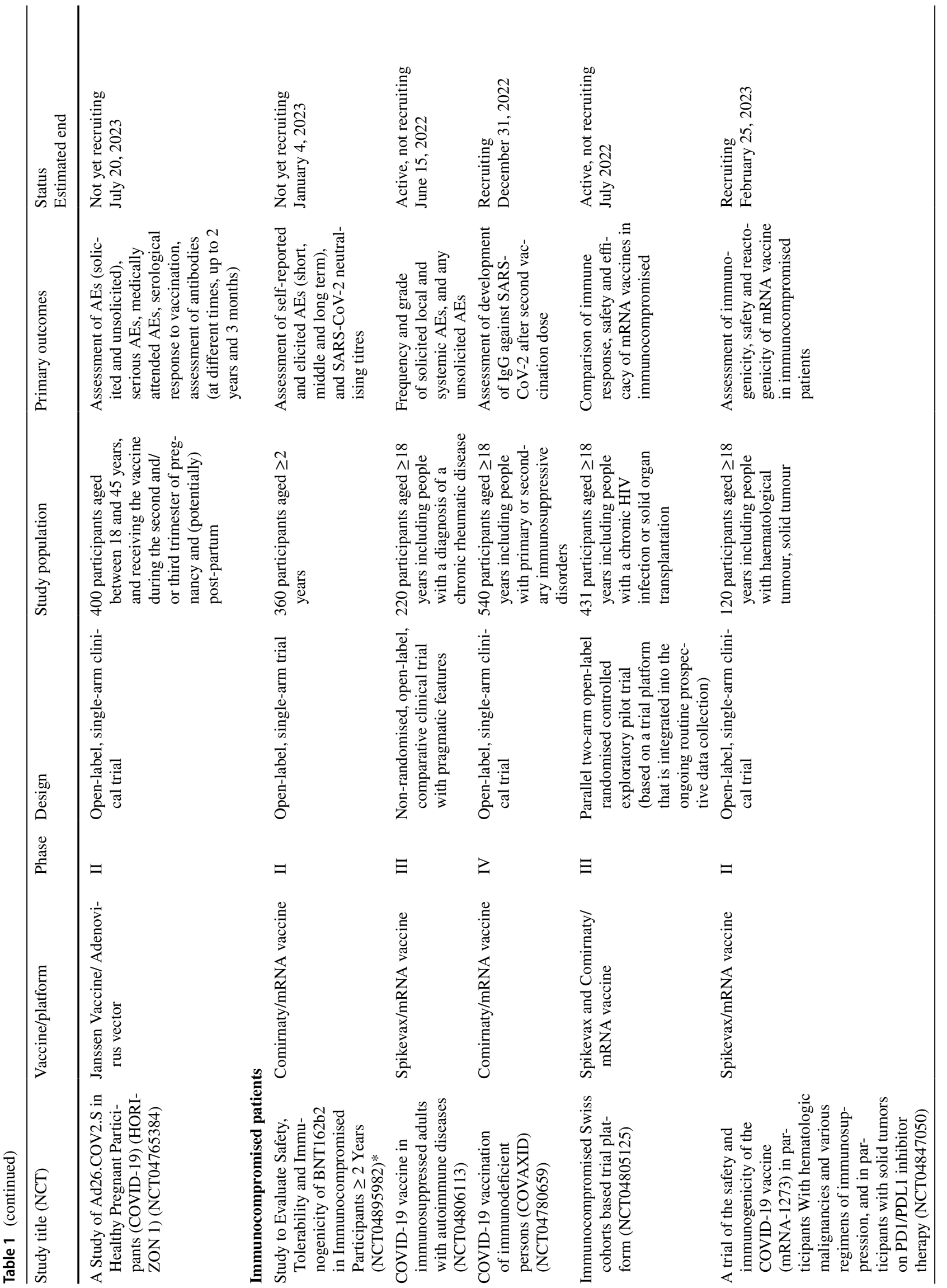




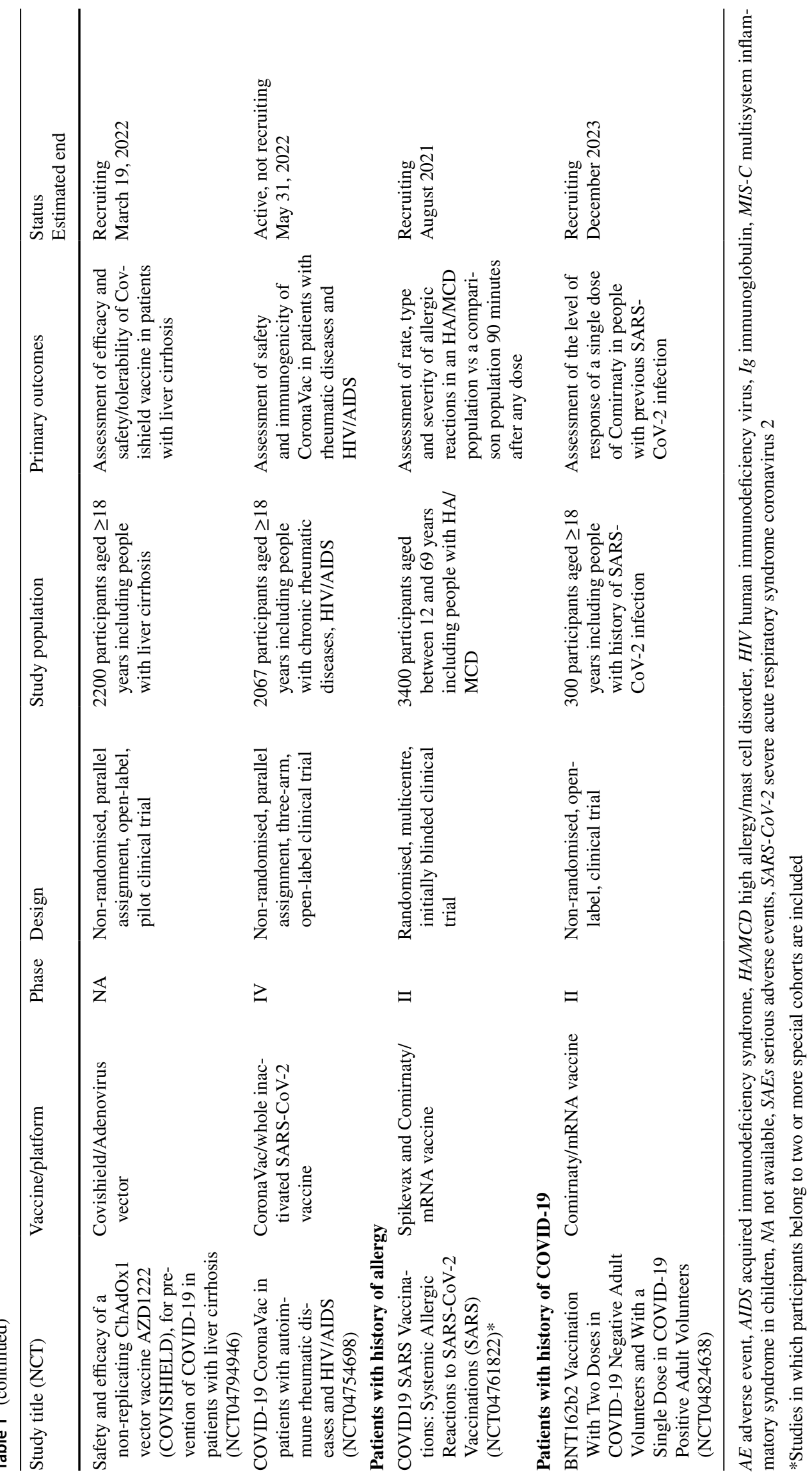


neonates have been shown to be more vulnerable to severe COVID-19 disease [20]. In addition, over the course of the pandemic, a rare paediatric multisystem inflammatory syndrome temporally associated with SARS-CoV-2 infection (PIMS-TS), defined as multisystem inflammatory syndrome in children (MIS-C) by the World Health Organization (WHO), has been reported. This condition includes different types of clinical manifestations such as Kawasaki-like symptoms and life-threatening shock [21].

Despite the asymptomatic or very mild symptomatic disease in children and adolescents, their role in transmission is not yet fully clarified. Transmission potential was shown in both children and adolescents, but children aged 0-9 years seem to be less likely to transmit the infection to a household member compared with adults, while adolescents seem to exhibit a higher potential [22]. Further research is needed to better understand transmission dynamics in the younger age classes.

During the different phases of the pandemic waves, and through the adoption of different testing strategies (e.g. testing asymptomatic individuals and population screening), the easing of mitigation measures (specifically the reopening of schools) could be linked to the increase in COVID-19 cases among children and adolescents as a result of the improved case ascertainment. In Italy, the rate of cases in children and adolescents increased from $1.8 \%$ during the lockdown (March-May 2020) to 8.5\% during the post-lockdown phase (June-September 2020) [23]. Although children and adolescents still account for a minority of cases, an increased proportion of reported cases has been observed also in other EU/EAA countries, especially since January 2021 [24]. In addition, to fully understand the epidemiology of SARSCoV-2 infection in children and adolescents, it is essential to take into account the changing context due to the emergence of variants of concern that usually show a greater transmissibility across all age groups. A study conducted in the context of high prevalence of the Alpha (B.1.1.7) variant found no evidence of more severe disease in children and young people, suggesting that severe acute respiratory COVID-19 still represents an uncommon circumstance in children and young people [25]. Considering the rapid spreading of the Delta (B.1.617.2) variant that has become prevalent in all European countries, even more transmissible than previous strains, the high risk of concentrated circulation in the paediatric and adolescent population less covered by the vaccine campaigns should not be overlooked.

\subsubsection{Pre-Marketing Evidence}

Only a few data on safety and efficacy of COVID-19 vaccines in children and adolescents have been published so far, while more than 20 trials on 15 different vaccines are ongoing (Table 1).
The paediatric population is usually enrolled in clinical trials after evidence of a positive benefit-risk profile in adults and this is the case with some of the COVID-19 vaccines, which have been firstly tested on adults (from 16 years old) and then further extended to lower ages.

Results of a pivotal trial including 2260 participants aged between 12 and 15 years led to the approval of Comirnaty, the first approved COVID-19 vaccine in Western countries for individuals aged $<16$ years. It demonstrated non-inferiority compared with results in younger adults, both in terms of antibody titres and infection rate, as well as a reassuring safety profile $(<0.4 \%$ of study participants experienced severe but rapidly solved vaccine-related adverse events after the first or second dose) [26]. In July 2021, the European Medicines Agency (EMA) also granted an extension of indication for the COVID-19 vaccine Spikevax in children aged between 12 and 17 years [27].

Results of a pre-marketing study on adolescents and children are also available in the literature for CoronaVac (by Sinovac). This is a whole, inactivated vaccine currently marketed by many Asian, Southern American and African countries for adults. The vaccine has also been approved for use in younger people in some of these regions (first approved by China and Indonesia) [28]. The key trial recruited about 500 subjects and found $93 \%$ of seroconversion after the second dose, with better results for the higher dose group. Only two cases of grade 3 adverse events ascribed to vaccination were observed [29]. All published data only represent results from ad-interim analysis since the follow-up is planned to continue for up to $1-2$ years.

Children/adolescents aged under 16 years are usually involved in phase II trials, with a few exceptions, with aged 12 years and older being the most frequently represented age class; some studies also enrolled participants starting from 6 months of age. Randomised placebo-controlled trial is a very frequent design. The assessment of adverse events is the most frequently listed primary outcome in all trials, followed by short-term antibody titre detection (either neutraliser only or also anti-S). In a few studies, a follow-up of 1-2 years is planned, and clinical efficacy (incidence of severe COVID19 symptoms) is also included among secondary outcomes.

\subsubsection{Post-Marketing Evidence}

The post-approval phase will be particularly critical in the overall risk-benefit assessment of COVID-19 vaccines for children/adolescents. On one hand, vaccine effectiveness in the pre-marketing evaluation was assessed through 'immunobridging', namely inferring from immunogenicity data from older populations. Specifically, the primary basis for the establishment of efficacy in 12- to 15-year-old adolescents was a neutralizing antibody response that was found to be non-inferior to that in vaccine recipients 16 years of 
age or older, for whom efficacy had been shown [26]. On the other hand, safety data were only obtained through 1 month of follow-up after dose 2 for some participants, thus making proactive vaccinovigilance pivotal for the actual real-time appraisal of tolerability and effectiveness of COVID-19 vaccines in this 'paediatric cohort'.

As regards safety assessment, establishing background rates of adverse events of special interest (AESI) [30] by age, sex and race/ethnicity is critical to understand and identify real vaccine-related safety signals, including neurological, autoimmune and cardiovascular disorders [31]. These background rate data on 26 AESIs have been collected within the EMA-funded ACCESS (vACcine Covid-19 monitoring ReadinESS) project [32].

Myocarditis/pericarditis was recognised as a rare complication of COVID-19 mRNA vaccinations, especially in young adult and adolescent males. According to the Centers for Disease Control and Prevention (CDC), myocarditis/ pericarditis reporting rates are approximately 12.6 cases per million doses for the second dose of an mRNA vaccine among 12- to 39-year-olds, with higher reporting rates among males aged $12-17$ years and those aged $18-24$ years (62.8 and 50.5 reported myocarditis cases per million second doses of mRNA COVID-19 vaccine administered, respectively) [33]. Based on the latest comprehensive review, also collecting published cases reports, patients with myocarditis invariably presented with chest pain, usually 2-3 days after a second dose of mRNA vaccination, and had elevated cardiac troponin levels, with ST segment elevations on the electrocardiogram in most of the cases, and cardiac magnetic resonance imaging (MRI) suggestive of myocarditis in all tested patients [34]. Monitoring of myocarditis/pericarditis events in such a population is crucial and identifying these conditions promptly is necessary to avoid unnecessary and expensive investigation in these patients. However, its pathophysiology has not been determined yet and the causal association between the mRNA vaccines and myocarditis is still unknown.

In one case, a cardiomyopathy gene panel was negative, but autoantibody levels against certain self-antigens and frequency of natural killer cells were increased. The reasons for male predominance in myocarditis cases are unknown, but sex hormone differences in immune response and myocarditis, as well as potential under-diagnosis of cardiac disease in women should be explored. While various underlying mechanisms have been hypothesised, almost all cases were reversible with or without treatment; in addition to supportive care, nonsteroidal anti-inflammatory drugs, steroids, and colchicine were used in some of the patients. A few patients were treated with intravenous immunoglobulin and acetylsalicylic acid, and some were initiated on $\beta$-blocker and angiotensin-converting enzyme inhibitor therapy due to left ventricular systolic dysfunction. As an initial evaluation, electrocardiogram cardiac troponin level should be obtained (plus inflammatory markers), with cardiology consultation and evaluation though echocardiography and cardiac MRI in suspected cases.

\subsubsection{Recommendation for COVID-19 Vaccination}

As mentioned above, based on the positive evaluation of the available safety and efficacy data for this population, Comirnaty approval for use was expanded to young adolescents (12-15 years) by the Food and Drug Administration (FDA) on May 10, 2021 [26], and later on by the EMA on May 28, 2021 [35]. On July 23, 2021, EMA's human medicines committee (CHMP) also recommended an extension of indication for Spikevax to include use in children aged 12-17 years [27].

However, debate exists about the actual benefit-risk profile of COVID-19 vaccines in children/adolescents, with some experts pointing out several critical issues to be specifically considered in the paediatric population. First, COVID-19 complications, such as hospitalisation, severe illness and death, as well as SARS-CoV-2 infection susceptibility are significantly lower in children/adolescents than in adults [5]. As such, COVID-19 vaccinations may have less direct protective effects in the paediatric population than in adults, with marginal benefits in protecting others, especially people at higher risk, who have already been immunised [36, 37]. Second, some believe that as there are currently very few (long-term) safety data available in this population, it is difficult for policymakers to make informed decisions on whether children should receive COVID-19 vaccines.

On the contrary, other authors promote the vaccine uptake in adolescents, as quickly and equitably as possible, by underlying the increasing evidence on the potential role of vaccination to protect the whole society, including older adults, and to decrease household transmission of SARS$\mathrm{CoV}-2$ infection. Current evidence suggests that short-term severe consequences of COVID-19 are considerably less common in children than in older adults, and in children the risk of long-term COVID-19 negative effects (i.e. long covid, multisystem inflammatory, or Kawasaki disease) is greater than potential risks associated with COVID-19 vaccination [38-41].

The US CDC recommends everyone 12 years and older should get a COVID-19 vaccination, to help protect against COVID-19, as a critical strategy to stop the pandemic. The agency also supports the co-administration of COVID-19 vaccines and other routine paediatric vaccines [42].

As the course of COVID-19 disease is typically milder in healthy adolescents, the European Centre for Disease Prevention and Control (ECDC) recommends giving priority in COVID-19 vaccination to older age groups before targeting adolescents. Another recommendation is prioritizing the 
vaccine uptake for teenagers that are at high risk of severe COVID-19 in the same way as for all people at high risk of severe disease in other age groups. To support further decision making on vaccination of adolescents, the need for continuous monitoring of the spread of variants among younger individuals and assessment of the long-term effects of COVID-19 has been also highlighted by ECDC [43].

The Medicines and Healthcare products Regulatory Agency (MHRA) evaluated emerging cases of rare but serious adverse events following the use of mRNA vaccines reported from the UK and other countries, including myocarditis and pericarditis, predominantly in young male adults. Accordingly, recommendations from the UK Joint Committee on Vaccines and Immunisation (JCVI) advise that only children and young people aged 12 years of age and older with specific underlying health conditions for which they are considered at increased risk for serious COVID-19, such as severe neuro-disabilities, Down's syndrome, immunosuppression, and severe learning disabilities, should be offered COVID-19 vaccination [13].

Another issue is that worldwide laws require parental permission for vaccination, by presuming that parents (or guardians) would make a decision in the best interest of their children. Nevertheless, parents and minors might disagree about healthcare intervention, mainly in the context of this pandemic, where adolescents and some older children may have a different perceptions of risk and benefits of COVID19 vaccination than their parents. Accordingly, some authors suggested an age grouping for minor permission rules (i.e. younger than 12 years, aged 12-14 years, and aged 15-17 years, according to their ability to judge the intervention) to support states in the recognition of the minors to consent to COVID-19 vaccination even without parental permission [44].

\subsection{Pregnant and Lactating Women}

\subsubsection{Epidemiology of SARS-CoV-2 Infection}

Over the course of the COVID-19 pandemic, publications on COVID-19 in pregnancy drastically increased, from individual case reports and case series to observational studies and systematic reviews [45]. Based on the current knowledge, pregnant women do not seem to be at higher risk of becoming infected with SARS-CoV-2, that is, they do not seem to be more susceptible to the virus than people who are not pregnant in the same context [46].

However, it has been recently shown that pregnant women are at higher risk for severe illness from COVID-19, once infected. According to the CDC, in the period from January 2020 to July 2021, a total of 101,710 pregnant women were diagnosed with COVID-19 in the US, of whom $448(0.44 \%)$ were admitted to an ICU and $114(0.11 \%)$ died (mostly among ethnic minorities, i.e. non-White) [47]. Moreover, in a study of about 400,000 women aged 15-44 years with symptomatic COVID-19, compared with non-pregnant women, pregnant women were more likely to be admitted to an ICU, receive invasive ventilation and extracorporeal membrane oxygenation (ECMO), and had $70 \%$ increased risk of death [48]. This is in line with a series of reports published during the last months of 2020 [45].

Regarding perinatal outcomes following SARS-CoV-2 infection, several reports of miscarriage and stillbirth were published [49, 50], as well as pathological findings such as placentitis [51] and placenta thrombosis [52]. Premature births have been shown to be more common in SARS-CoV2-positive mothers and their newborns are more likely to be admitted to the neonatal care unit [45]. A significant association of preeclampsia, gestational diabetes and low birth weight and SARS-CoV-2 infection in pregnant women was also identified [53].

Consistently, mothers' mental health is also at risk: women who are pregnant during the pandemic are significantly more stressed and worried than before [54] and they may develop symptoms of post-traumatic stress disorder, particularly if previously suffering from anxiety or depression [55].

\subsubsection{Pre-Marketing Evidence}

Pregnant and breastfeeding women were not included in any pre-marketing trial for COVID-19 vaccines, so premarketing evidence on these categories is not available so far. Preclinical studies of animals receiving a COVID-19 vaccine before or during pregnancy did not raise any safety concerns [56].

The first vaccine trial in pregnant women began in February 2021 (ClinicalTrials.gov identifier NCT04754594). It is an ongoing phase II/III randomised, placebo-controlled, observer-blinded study of 700 healthy pregnant women, 18 years of age or older, vaccinated during 24-34 weeks of gestation. The study will evaluate safety, tolerability and immunogenicity of two doses of Comirnaty vs placebo, administered 21 days apart. The study will also assess the transfer of potentially protective antibodies to infants, who will be monitored for safety through approximately 6 months of age.

A phase II clinical study is also planned, but not yet recruiting, to evaluate safety, reactogenicity and immunogenicity of COVID-19 Vaccine Janssen in healthy pregnant participants (NCT04765384).

\subsubsection{Post-Marketing Evidence}

In the absence of pre-marketing data, post-marketing evidence of efficacy and safety of COVID-19 vaccines in pregnancy is of paramount importance. 
A retrospective cohort study conducted in Israel, using a large registry of 15,060 pregnant women, reported an adjusted hazard ratio for SARS-CoV-2 infection of 0.22 (95\% CI 0.11-0.43) with Comirnaty vaccination versus nonvaccination [57], highlighting the protective effect of the vaccine against SARS-CoV-2 infection in vaccinated versus unvaccinated pregnant women.

In an observational study of 122 pregnant women (gestational age 35-41 weeks), COVID-19 mRNA vaccines were found to induce a robust maternal immune response, with transplacental antibody transfer detectable in cord blood as early as 16 days after the first dose [58], thus suggesting protection of the newborn.

A cohort study of 131 reproductive-age vaccine recipients (84 pregnant, 31 lactating and 16 nonpregnant women) showed that vaccine-induced antibody titres were equivalent in pregnant and lactating women compared with nonpregnant women and also the presence of antibodies in breastmilk of women immunised with COVID-19 mRNA vaccines. Moreover, no safety issue emerged from this study [59].

Consistently, a prospective cohort study of 84 breastfeeding women who received COVID-19 mRNA vaccine found robust secretion of SARS-CoV-2-specific immunoglobulin (Ig) A and $\operatorname{IgG}$ antibodies in breast milk for 6 weeks after vaccination. Antibodies found in breast milk showed strong neutralizing effects, suggesting a potential protective effect against infection in the infant [60].

In addition, a number of US [61] and European active pharmacovigilance projects and registries on COVID-19 vaccine safety during pregnancy and breastfeeding are ongoing. Preliminary findings from the v-safe registry did not show safety signals among pregnant women who received COVID-19 mRNA vaccines; among 3958 participants, 115 (13.9\%) resulted in a pregnancy loss [62]. Even if not directly comparable, proportions of adverse pregnancy events were similar to those from studies conducted before the COVID-19 pandemic [62]. Indeed, according to the American College of Obstetricians and Gynecologists (ACOG), in the general population, the frequency of clinically recognised early pregnancy loss for women aged between 20 and 30 years is $9-17 \%$, and it sharply increases with increasing age (up to $80 \%$ at 45 years of age) [63].

\subsubsection{Recommendation for COVID-19 Vaccination}

Although not always explicitly recommended, COVID-19 vaccination is currently indicated in pregnancy and breastfeeding by most scientific societies and regulatory agencies, including the ACOG [64], the Royal College of Obstetricians and Gynaecologists (RCOG) [65], the Royal Australian and New Zealand College of Obstetricians and Gynaecologists (RANZCOG) [66], CDC [56] and the Italian Society of
Obstetrics and Gynaecology (SIGO) [67]. WHO currently recommends vaccination in pregnant women "when the benefits of vaccination to the pregnant woman outweigh the potential risks" (e.g. women at high risk of exposure or at high risk of developing severe COVID-19, such as having comorbidities), while recommends vaccination in breastfeeding women as in other adults [68].

There is currently no preference for the use of one COVID-19 vaccine over another; however, pregnant women aged $<18$ years are currently only eligible to receive Comirnaty or Spikevax because only mRNA vaccines are authorised for people aged under 18 years.

COVID-19 vaccines have not been studied in breastfeeding women but, based on their mechanism of action, currently available vaccines are thought not to carry any risk to lactating women or their breastfeeding babies. Therefore, there is no need to avoid initiation or discontinue breastfeeding in patients who receive a COVID-19 vaccine.

Finally, for those attempting to become pregnant, there is no evidence that COVID-19 vaccines affect fertility, and vaccination should not be postponed [69].

The limited evidence on the use of COVID-19 vaccines in pregnancy and breastfeeding highlights the need for further studies on this topic.

\subsection{Immunocompromised Patients}

\subsubsection{Epidemiology of SARS-CoV-2 Infection}

Evidence on COVID-19 in a wide range of immunocompromised patients is rapidly increasing.

A number of observational studies have documented that patients with solid tumours [70, 71] or hematologic malignancies [72-75], solid organ transplant recipients [76], hematopoietic cell transplant recipients [77] and patients with human immunodeficiency virus (HIV) and primary immunodeficiency are at higher risk of developing COVID-19-related severe outcomes [78, 79]. A recent systematic review and meta-analysis of both experimental and observational studies showed that in patients with autoimmune diseases the risk of COVID-19 was significantly higher than in control patients (odds ratio $2.19,95 \% \mathrm{CI}$ $1.05-4.58 ; p=0.038$ ) [80]. Concerning drugs used to treat such diseases, chronic use of glucocorticoids (especially at high doses), but not disease-modifying anti-rheumatic drugs (DMARDs), have been associated with a substantially higher risk of severe COVID-19-related outcomes [80-82]. Conversely, evidence on the effects of antineoplastic chemotherapy on COVID-19 outcomes is controversial; while one observational study showed that recent chemotherapy treatment (i.e. therapy administered within 1 month before testing positive for SARS-CoV-2 infection) was not associated with adverse COVID-19 outcomes [83], a more recent 
observational study documented that recent chemotherapy was associated with higher COVID-19 severity and 30-day mortality [84].

\subsubsection{Pre-Marketing Evidence}

Overall, six ongoing clinical trials aimed to assess COVID19 vaccines' immunogenicity and safety in immunocompromised patients were identified. Of these, one was a phase II clinical trial (NCT04847050), two were phase III clinical trials (NCT04806113; NCT04805125), two were phase IV clinical trials (NCT04780659; NCT04754698) and one was not specified (NCT04794946). Two of these trials concerned two vaccines not authorised in the European Union-Covishield (NCT04794946) and CoronaVac (NCT04754698). Overall, 4927 immunocompromised patients are expected to be enrolled in those ongoing clinical trials from the US, Europe, South America and India (NCT04847050; NCT04780659; NCT04754698; NCT04794946). In addition, 651 immunocompromised patients have been enrolled in clinical trials that are ongoing in Canada and Switzerland (NCT04806113; NCT04805125). First results are expected starting from 2022.

\subsubsection{Post-Marketing Evidence}

Due to high risk of severe SARS-CoV-2 infection, immunocompromised patients have been prioritised for COVID-19 vaccination, especially in Western countries, where they are almost fully vaccinated at this time. The lack of pre-marketing evidence in these patients signals the need for urgent real-world evidence generation of a vaccine benefit-risk profile in immunocompromised patients.

An increasing body of evidence coming from real-world studies shows that COVID-19 vaccination with Comirnaty vaccine in transplant recipients is associated with impaired responses.

An observational study conducted by Boyarsky et al. found that among 658 solid organ transplant (i.e. kidney, lung, pancreas, heart, liver) recipients, 301 (46\%) had no antibody response following dose 1 or 2 of SARS-CoV-2 mRNA vaccines [85]. Also, a reduced seroconversion rate was observed after one vaccination dose in patients with haematological cancer [86], kidney transplant [87, 88] and patients treated with immunosuppressants $[86,89]$; thus, the advice is not to delay the second dose beyond 21 days from the first vaccination dose [90].

A reduced immune response was also observed after two doses of COVID-19 vaccine in patients with solid and haematological cancer [91, 92] and in transplant recipients [93]. Moreover, an observational study of 40 vaccinated kidney transplant recipients showed that, after the second dose of
Comirnaty, none of the vaccinees developed comparable IgG titres to healthy controls [94]. Similarly, a real-world study comparing the antibody responses of 48 vaccinated lung transplant recipients with 33 lung transplant recipients with prior SARS-CoV-2 infection reported that none of the vaccinees tested after two doses of Comirnaty developed anti-SARS-CoV-2 IgG, while $85 \%$ of patients with history of SARS-CoV-2 infection presented an antibody response [95].

Immunosuppressant therapy in solid organ transplant recipients is a key factor inhibiting the humoral response to the COVID-19 vaccine. Furthermore, real-world data concerning vaccinated dialysis patients suggest that in these patients the post-vaccination humoral response may be delayed and/or reduced by several factors related to the uremic condition [96].

\subsubsection{Recommendation for COVID-19 Vaccination}

Although evidence on safety and efficacy of COVID-19 vaccines in immunocompromised patients is still sparse, the use of COVID-19 vaccines in this category of patients is recommended due to the high risk of having severe SARS-CoV-2 infection [97-100]. Considering evidence on the reduced effectiveness of COVID-19 vaccines in immunocompromised patients, the administration of a third dose is being considered [101, 102]; accordingly, two ongoing clinical trials are currently investigating the efficacy and safety of a third dose in these patients (NCT04895982; NCT04885907). On the other hand, an observational study showed that, as compared with the first dose of Comirnaty and Vaxzevria, the second dose was associated with an increased effectiveness $(73 \%, 95 \%$ CI $33.9-89.0$ and $74.6 \%, 95 \%$ CI $18.7-92.1$, respectively) in immunocompromised people [103], thus questioning the real need for a third dose within a short interval.

In addition, immunosuppressive drug use could affect the production of neutralizing antibodies [104], thus some scientific societies suggested that the timing of administration of some immunosuppressive drugs (e.g. rituximab and abatacept) should be rescheduled to maximise vaccine response [105-107]. For instance, in patients on chronic treatment with rituximab, the first dose of a COVID-19 vaccine should be given 4 weeks before the next scheduled rituximab cycle; after the second vaccination dose, if disease is well controlled, rituximab treatment should be delayed for 2-4 weeks. In the case of subcutaneous chronic treatment with abatacept, patients should discontinue treatment 1 week before and 1 week after the first vaccination dose; instead, in patients receiving intravenous abatacept, the first vaccination dose should be administered 4 weeks after abatacept infusion, while subsequent abatacept infusion should be delayed by 1 week. 


\subsection{Patients with History of Allergy}

\subsubsection{Epidemiology of SARS-CoV-2 Infection}

Allergic diseases include food allergies, rhinitis, conjunctivitis, angioedema, urticaria, eczema, eosinophilic disorders, drug/biological agent hypersensitivity, insect allergies, occupational allergies and asthma [108, 109]. The World Allergy Organization estimates that between 10 and $40 \%$ of both adults and children have at least one of these allergic diseases [109], with asthma alone affecting 262 million people in 2019 and 461,000 deaths globally [110].

It is still not clear whether allergic diseases are associated with a higher risk of testing positive for SARS-CoV-2 or severe clinical outcomes from COVID-19, as most of the allergic diseases were not considered during the studies conducted on patients affected by COVID-19 and their comorbidity-related risks. In this regard, asthma is the allergic disease for which there are currently more data in the literature. A systematic review and meta-analysis based on 119 studies including a total of 403,392 cases showed that patients with COVID-19 had a pooled prevalence of asthma similar to that of the general population $(8.3 \%$ vs $4.3-8.6 \%$, respectively) and, therefore, asthma may not be associated with an increased risk of COVID-19. The meta-analysis was conducted in 116 of the 119 included studies and did not show a higher risk of poor COVID-19-related outcomes [111].

Regarding other types of allergies, a Korean cohort study of 219,959 patients found that individuals affected by allergic rhinitis were more likely to test positive for SARS-CoV-2 (adjusted OR 1.18; 95\% CI 1.11-1.25) and have worse COVID-19-related clinical outcomes, such as intensive care admission or death (adjusted OR 1.27; 95\% CI 1.00-1.64). Conversely, those with atopic dermatitis were not identified to be at higher risk of susceptibility to SARS-CoV-2 infection or severe clinical outcomes [112].

\subsubsection{Pre-Marketing Evidence}

Only few cases of severe allergic reactions or anaphylaxis to COVID-19 vaccines have been reported in the pre-marketing studies. This is not surprising considering the overall very low incidence of anaphylaxis and, more importantly, the exclusion of individuals with a history of severe hypersensitivity reactions in most pre-marketing studies. Specifically, patients with previous history of severe allergic reactions associated with any vaccine and subjects with severe allergic reaction to any component of COVID-19 vaccine were excluded from the Comirnaty pivotal trial (NCT04368728); the latter were also excluded from the Spikevax pivotal trial (NCT04283461). In general, history of allergy (anaphylaxis) was listed as the main exclusion criterion in all the protocols of the pivotal clinical trials for the vaccines currently approved by EMA as of July 28, 2021. Only one ongoing clinical trial (NCT04761822) on COVID-19 vaccines was identified that includes people with history of allergy and aims to assess systemic allergic reactions to COVID-19 mRNA vaccines in such a population.

\subsubsection{Post-Marketing Evidence}

On December 8, 2020, the UK initiated vaccination with Comirnaty and two women developed immediate anaphylaxis within $24 \mathrm{~h}$. After the review of additional data, on December 30, 2020, the MHRA included a contraindication of use in people with previous history of allergic reactions to the ingredients of the vaccine, but not in those with any other allergies [113].

As for the incidence of allergic reaction after COVID-19 vaccine administration, a first CDC report of the US Vaccine Adverse Event Reporting System (VAERS) database reported 21 cases of anaphylaxis out of 1,893,360 first doses (estimated rate of 11.1 cases per million doses administered) of Comirnaty during December 14-23, 2020. Around 70\% of vaccinees experiencing anaphylaxis had symptom onset within 15 minutes from vaccination [114].

Similarly, the CDC showed that 10 cases of anaphylaxis were reported after administration of the Spikevax vaccine during the period December 21, 2020 to January 10, 2021, resulting in a rate of 2.5 cases per million doses administered [115].

An updated analysis of VAERS through January 18, 2021, reported rates of 4.7 and 2.5 cases of anaphylaxis per million doses administered of Comirnaty and Spikevax, respectively [116]. A recent observational study based on self-reported data showed a rate of severe reactions consistent with anaphylaxis, defined according to the Brighton criteria, following COVID-19 vaccination corresponding to 2.47 per 10,000 doses. Specifically, $10(63 \%)$ and $5(31 \%)$ of the 16 subjects experiencing anaphylaxis had previous history of allergy and anaphylactic reactions, respectively [117].

Following the extended use of COVID-19 vaccines worldwide, several cases of severe allergic reactions, in particular with mRNA vaccines, were collected through spontaneous reporting systems, and the US FDA and EMA introduced, as a contraindication to use of mRNA vaccines, a past history of hypersensitivity (e.g. anaphylaxis) to any component of these vaccines. In any case, a second dose of the COVID-19 vaccines should not be given to those who have experienced anaphylaxis or severe allergic reactions to the first dose. A recent multicentre observational study conducted in the US by Krantz et al. corroborates the safety of the second dose of mRNA COVID-19 vaccines in subjects who reported immediate suspected allergic reactions after the first dose (except for subjects with severe allergic 
reactions and/or evidence of IgE-mediated allergy), based on a previous allergist assessment [118].

A debate about the potential increase of risk of severe allergic or anaphylactic reactions following COVID-19 vaccination among individuals with a history of allergy has been raised in the scientific community. At first, the enhanced surveillance of over one million doses of the vaccine in the UK and North America found no evidence of an increased risk of anaphylaxis in those with prior severe but unrelated allergic reactions [119].

Among the 66 case reports of anaphylaxis received in US VAERS from December 14, 2020, to January 18, 2021 [116], $21(32 \%)$ mentioned a prior episode of anaphylaxis and $52(79 \%)$ included a documented history of allergies or allergic reactions derived from exposures to other substances (e.g. other vaccines, contrast media, sulfa drugs, penicillin, prochlorperazine, latex, walnuts, unspecified tree nuts, jellyfish stings, etc.). Most anaphylaxis cases had symptom onset within $30 \mathrm{~min}$ of vaccination and occurred in women.

More research is needed to identify the cause of the potentially increased rate of anaphylaxis to COVID-19 vaccines, but polyethylene glycol (PEG) 2000, an excipient used to cover the lipid nanoparticles (LNSs) that contain the mRNA in the COVID-19 mRNA vaccines, is deemed to be the possible culprit of anaphylactic reactions [120].

Although the mechanism of PEG-induced hypersensitivity is not exactly clear, IgE, IgM and IgG antibodies against PEGs are believed to be involved in this immune reaction. It has been demonstrated how the different PEG types are not all equally capable of causing allergic reactions, while the risk appears to be dependent on molecular weight, route of administration (i.e. intramuscular or intravenous route) as well as cross reactivity with polysorbate [121, 122].

\subsubsection{Recommendation for COVID-19 Vaccination}

Public Health England (PHE) and CDC, in accordance with The American College of Allergy, Asthma and Immunology (ACAAI) and the European Academy of Allergy and Clinical Immunology (EAACI), currently recommend that everyone should be offered COVID-19 vaccination unless there is a suspect or confirmed allergy to a component in the COVID-19 vaccines for which a detailed assessment from a specialist/allergologist may be required [123-126]. Moreover, those with history of anaphylaxis following the first dose of any COVID-19 vaccine are advised not to receive a second dose of the same vaccine, and a specialist's evaluation should be recommended [124].

Due to the rarity of severe allergic reactions to COVID-19 vaccination and the not yet elucidated mechanisms underlying those adverse reactions, diagnostic procedures to predict allergic reactions to COVID-19 vaccines are not consistently recommended [120,127]. Because of the hypothesis of a potential contribution of PEG in allergic reactions to COVID-19 mRNA vaccines, some individuals may be offered skin testing for PEG sensitisation before vaccination [120].

Nevertheless, according to a recent systematic review of several clinical guidelines, testing for PEG sensitisation should not be done routinely, as its sensitivity/specificity is not yet known and the evidence regarding the potential of the testing in predicting severe allergic reactions is low. Generally, allergic patients should get vaccinated in a setting in which the health-care provider is trained to manage any potential life-threatening event and where life-saving devices and drugs (such as epinephrine) are immediately available. Moreover, vaccinees should be routinely observed for at least $15 \mathrm{~min}$ and for a longer observation time in vaccinees with history of severe allergy. Some individuals, such as those with mast cell disorders and with uncontrolled asthma, are advised to receive the vaccine only within a hospital setting for safety purposes. Furthermore, these individuals may be advised to take premedication such as antihistamines before getting vaccinated [128]. However, there is paucity of evidence that antihistamines or corticosteroids pre-medication can actually prevent anaphylaxis [129].

\subsection{Patients with History of COVID-19}

\subsubsection{Epidemiology of SARS-CoV-2 Reinfection}

A population-based study of 525,339 subjects who received a swab during the first wave of the COVID-19 pandemic of 2020 with 11,707 positives, reported reinfection in 72 subjects during the second wave, compared with 16,819 positive subjects in the second wave out of 514,271 who were negative in the first wave (rate ratio [RR] 0.65; 95\% CI 0.51-0.82) [130]. The estimated protection from infection after about 6 months is $80.5 \%$ (95\% CI 75.4-84.5). However, protection drops to $47.1 \%$ (95\% CI 24.7-62.8) in subjects over the age of 65 years. Of note, there are no relevant differences in gender or in the duration of protection over time (3-6 months vs $>7$ months).

These results sparked a debate on the advisability of vaccinating subjects with a previous history of SARS-CoV-2 infection on the basis of an individual and population risk-benefit profile that should take into account several variables. There are some questions that require urgent answers to guide clinical decisions.

In the first place, it is feared that the protection in subjects immunised from the disease is shorter than that obtained with vaccination, although reassuring information are available for the short term.

Secondly, the duration of immunity linked to the infection seemed to vary with age. In elderly subjects, a dose of the vaccine after recovery from the disease, although it 
is not clear in what timeframe, could guarantee important benefits, even without a second dose. In others, there are no appreciable benefits from vaccination and therefore the vaccination-related risks should be avoided.

Third, the findings of the above-mentioned studies are related to periods in which the currently dominant SARS$\mathrm{CoV}-2$ variants did not circulate; as such, it is possible that the benefit of vaccination in a subject with immunity gained from previous infection with a specific SARS-CoV-2 variant changes in relation to the spread of different variants of the virus becoming dominant. Finally, it is not certain that the benefit received from the different vaccines (e.g. viral vector or mRNA) is the same. Many of these questions will be answered in ongoing post-authorisation studies.

\subsubsection{Pre-Marketing Evidence}

In all pre-marketing studies of all COVID-19 vaccines, the history of previous COVID-19 infection is listed among the main exclusion criteria. Only one ongoing clinical trial (NCT04824638) on COVID-19 vaccines aiming to assess the level of response of a single dose of Comirnaty in people with previous SARS-CoV-2 infection was identified.

\subsubsection{Post-Marketing Evidence}

Studies conducted on laboratory endpoints seem to confirm the benefits of a single dose in subjects with previous COVID-19. A recently published study described 63 COVID-19 convalescent subjects, of whom 23 (43\%) had received one or two doses of an approved mRNA vaccine [131]. In unvaccinated subjects, antibody reactivity to the receptor binding domain (RBD) of SARS-CoV-2, neutralizing activity and the number of RBD-specific memory $B$ cells remain relatively stable between 6 and 12 months after infection. In vaccinated subjects, there was an increase in all components of the humoral response and neutralizing activity against the variant of concern similar or superior to those observed against the original Wuhan Hu-1 strain obtained with vaccination.

Subsequent studies have not only confirmed these findings but have also documented that the improvement of immunisation in patients with history of COVID-19 obtained with the second dose is negligible compared with that obtained with a single dose $[132,133]$. Other studies conducted in India and the UK showed that the observed benefits of mRNA vaccines in convalescent subjects are similar, even with a single administration of a viral vector vaccine $[134,135]$.

Studies conducted on clinical outcomes are scarce. A study recently published as a pre-print [136] evaluated reinfection in a cohort of 52,238 Cleveland healthcare workers (vaccinated and unvaccinated with authorised mRNA vaccines). Among these, 2539 had previous SARS-CoV-2 infection. In a Cox proportional hazards regression model, vaccination was associated with a significantly lower risk of SARS-CoV-2 infection among those not previously infected (hazard ratio [HR] 0.031; 95\% CI 0.015-0.061) but not among those previously infected (none of the 1359 previously infected subjects who remained unvaccinated had a SARS-CoV-2 infection over the subsequent 6 months). This result seems to suggest that, at least in the short term, the protection offered against re-infection by the immunity developed by the infection itself is sufficient and does not benefit from vaccination. However, the risk of re-infection as well as COVID-19-related severe outcomes in relation to vaccination should also be evaluated in the long term.

To our knowledge, only one study [137] provided COVID-19 vaccine safety data in convalescent COVID19 subjects. This study, conducted in Saudi Arabia in 510 vaccinated subjects $(75 \%$ with Spikevax and $25 \%$ with Comirnaty) with a mean age of 25 years, did not observe statistically significant differences in the occurrence of adverse events following vaccination in subjects with prior COVID-19 infection as compared with subjects without prior infection.

\subsubsection{Recommendation for COVID-19 Vaccination}

There is a large body of evidence that people who have been infected with SARS-CoV-2 can benefit from vaccination, prompting the WHO and other public health agencies to recommend that such individuals be vaccinated. Some countries such as France, Germany and Italy recommend the use of a single dose of the vaccine in people with a previous SARS-CoV-2 infection (unless immunocompromised) in a defined timeframe in relation to the timing of the infection. This decision has probably also been influenced by the need to optimize the supply of vaccine, which could be intermittently available. In the US, where the vaccine is relatively abundant, two doses are recommended for those with prior SARS-CoV-2 infection [138].

\section{COVID-19 Vaccines Post-Marketing Surveillance}

The development of the new COVID-19 vaccines has been an example of the pandemic paradigm with overlapping phases, with shorter development time and faster assessment by regulatory agencies [139], while fulfilling all the requirements for efficacy and safety. However, some categories of people were initially excluded from pivotal clinical trials (e.g. pregnant women, children/adolescents and patients with allergy). 
The lack of pre-marketing evidence concerning the four COVID-19 vaccines authorised in Europe makes the role of spontaneous reporting of suspected adverse reactions to vaccines even more important to better understand the actual benefit/risk profile in a broader range of vaccinees. Active surveillance is pivotal to fully appreciate the tolerability profile in the real world [140]. In fact, numerous post-marketing surveillance programmes for COVID-19 vaccines have been funded both nationally and internationally.

A collaborative registry is advisable, as well as an active safety monitoring programme such as v-safe, a smartphonebased tool promoted by CDC, or the COVID Symptom Study app in the UK developed by health data company ZOE Global, proving particularly useful to collect real-world data on solicited events [141]. The assessment of efficacy and safety of vaccines in fragile subjects is also one of the aims of the ORCHESTRA project, which was mainly funded to follow long-term consequences of SARS-CoV-2 infections [142].

Likewise, the EMA funded an active pharmacovigilance project called 'Covid Vaccine Monitor' [143], preceded by other preparatory projects such as Early Covid Vaccine Monitor and Access, which is aimed at monitoring vaccine-related adverse reactions in several European countries, using a web-based app developed by the Netherlands pharmacovigilance centre Lareb. One of the main tasks of this project is to monitor the benefits and risks of different marketed COVID-19 vaccines through prospective webbased data collection in special cohorts (pregnant and lactating women, children/adolescents, immunocompromised, patients with a history of allergy and patients with previous SARS-CoV-2 infection) from eight European countries. The study protocol can be found on the European Network of Centres for Pharmacoepidemiology and Pharmacovigilance (ENCePP) website (register number: EUPAS42504).

Italy is participating in a large multidisciplinary network named 'ilmiovaccinocovid19 collaborating group', involving around 30 different centres including regional centres for pharmacovigilance, academic centres, public hospitals, local health units, scientific societies and representatives of patient associations, to help recruit vaccines in the monitoring programme through the use of the Italian version of the web-based app developed by Lareb (available at https:// www.ilmiovaccinocovid19.it).

\section{Conclusions}

Overall, the pre- and post-marketing evidence (whenever available) on the benefit-risk profile of currently EU-marketed COVID-19 vaccines on frailer populations or categories not included in pre-marketing trials is reassuring and in line with recommendations from scientific societies and regulatory agencies.

In addition to routine pharmacovigilance activities, in the near future, ongoing large-scale international prospective studies will provide clinically relevant data overall and more importantly in the frailest populations (e.g. pregnant and lactating women, children and adolescents, etc.) to better inform the worldwide COVID-19 vaccination campaign. Furthermore, although the COVID-19 pandemic concerns all countries around the world, significant differences in terms of vaccination rates and evidence generated on COVID-19 vaccination among developed and developing countries still exist.

Supplementary Information The online version contains supplementary material available at https://doi.org/10.1007/s40264-021-01131-6.

Acknowledgements IlmiovaccinoCOVID19 collaborating group: Gianluca Trifirò, Ugo Moretti, Nicoletta Luxi, Alexia Giovanazzi, Giuliana Petrelli, Silvia Girotti, Elena Arzenton, Lara Magro; University of Verona, Department of Diagnostics and Public Health Section of Pharmacology, Verona, Italy. Riccardo Lora, David Bellantuono, Alberto Sabaini, MedBrains. Alberto Firenze, Struttura Commissariale per l'Emergenza COVID della città metropolitana di Messina, Messina, Italy. Donatella Zodda, Fabrizia Guidotti, Maria Zappone, Bernardo Alagna, Messina Local Health Unit, Messina, Italy. Edoardo Spina, Paola Maria Cutroneo, Claudia Minore, Sicilian Regional Pharmacovigilance Centre, University Hospital of Messina, Messina, Italy. Claudio Costantino, Arianna Conforto, Francesco Vitale, University of Palermo, Palermo, Italy -Department of Health Promotion, Mother and Child Care, Internal Medicine and MedicalSpecialties "G. D'Alessandro", Hygiene section. Ilaria Morreale, Sicilian Regional Center of Pharmacovigilance, Azienda Ospedaliera Universitaria Policlinico P. Giaccone, Internal Medicine, Pharmacovigilance and Clinical Pharmacology Unit. Laura Marsala, Desirè Farinella, Silvana Bavetta, Azienda Ospedaliera di Rilievo Nazionale e di Alta Specializzazione (ARNAS) "Civico", Palermo, Italy. Emanuel Raschi, Elisabetta Poluzzi, Alma Mater Studiorum-University of Bologna, Italy - Dept. of Medical and Surgical Sciences. Maria Pia Fantini, Chiara Reno, Department of Biomedical and Neuromotor Sciences. Ester Sapigni, Anna Maria Potenza, Debora Podetti, Victoria Nikitina, Rita Ricciardelli, Nazanin Mogheiseh, Silvia Croce, Barbara Paltrinieri, Emilia-Romagna Pharmacovigilance Regional Centre, Bologna - Italy. Sofia Castellani, Elisa Sangiorgi, Margherita Selleri, Simona Lucchesi, Giuseppe Catucci, Ferrara Local Health Unit, Ferrara, Italy. Denis Savini, Chiara Sacripanti, Marco Faccioli, Maria Silvia Romio, Laura Rossi, Bologna Local Health Unit, Bologna, Italy. Simonetta Radici, Piacenza Local Health Unit, Piacenza, Italy. Giovanna Negri, Parma Local Health Unit, Parma, Italy. Lidia Fares, Reggio Emilia Local Health Unit, Reggio Emilia, Italy. Chiara Ajolfi, Modena Local Health Unit, Modena, Italy. Antonella Fadda, Antonella Chiarello, Imola Local Health Unit, Imola, Italy. Fabio Pieraccini, Francesco Pappalardo, Romagna Local Health Unit, Romagna, Italy. Marco Tuccori, Tuscany Region, Italy - Unit of Adverse Drug Reactions Monitoring, University Hospital of Pisa, Unit of Adverse Drug Reactions Monitoring, Pisa, Italy. Alfredo Vannacci, Roberto Bonaiuti, Claudia Ravaldi, Niccolò Lombardi, Giada Crescioli, PeaRL - Perinatal Research Laboratory, NEUROFARBA Department, University of Florence and CiaoLapo Foundation for Perinatal Health; Florence, Italy. Roberto Tessari, IRCCS Ospedale Sacro Cuore Don Calabria - Hospital Pharmacy, Negrar di Valpolicella, Italy. Emanuela Zandonà and Francesco Marchiori, University Hospital of Verona, Italy - Medical 
Coordination Unit. Cristiano Chiamulera, Unit of Clinical Pharmacology. Giovanna Zanoni, Gianenrico Senna, Unit of Immunology. Maria Angiola Crivellaro, University Hospital of Padua, Padova, Italy - Occupational Health Unit and Allergology Unit, Department of Cardiac Thoracic Vascular and Public Health Sciences University of Padova. Mauro Cancian, Departmental Allergy Unit. Sonia Faoro, Francesca Venturini, Pharmacy Department Padua, Italy. Marina Ferri, Luca Leonardi, Trento Local Health Unit, Trento, Italy. Sabrina Orzetti, Elisabetta Caccin, Paolo Baldo, Centro di Riferimento Oncologico (CRO) di Aviano, IRCCS - Pharmacy Unit, National Cancer Institute, Aviano, Italy. Annalisa Capuano, Concetta Rafaniello, Carmen Ferrajolo, Regional Centre of Pharmacovigilance and Pharmacoepidemiology, Naples, Italy. Claudia Pagliaro, Mariangela Mercaldo, Annamaria Fucile, Annalisa di Giorgio, Michele Tari, Sonia Manna, Giuseppina Farina, Cristina Di Mauro, Caserta Local Health Unit, Caserta, Italy. Ilenia De Carlo, Ilenia Senesi, Abruzzo Region, Pharmacovigilance Regional Centre, Italy. Claudia Pileggi, Caterina Palleria, Luca Gallelli, Caterina De Sarro, Giovambattista De Sarro, Department of Health Sciences, University of Catanzaro "Magna Græcia"/Regional Centre for Pharmacovigilance of Calabria, Catanzaro, Italy. Luigia Trabace, Mariagrazia Morgese, Stefania Schiavone, Paolo Tucci, Maria Bove, University of Foggia, Foggia, Italy. Francesco Lapi, Claudio Cricelli, Italian Society of General Practitioners. Giorgio Racagni, Italian Society of Pharmacology. Silvia Tonolo, Association of Patients with Rheumatic diseases. Eugenio Leopardi, Unione Tecnica Italiana Farmacisti (UTIFAR). Giusi Fava, Sandro Giuffrida, Vincenza Amato, Reggio Calabria Local Health Unit, Reggio Calabria, Italy. Marco Gambera, Valentina Montresor, Ospedale Pederzoli - Hospital Pharmacy, Peschiera del Garda, Italy.

\section{Declarations}

Funding No sources of funding were used to prepare this article.

Conflicts of interest Gianluca Trifirò has served in the last three years on advisory boards/seminars funded by SANOFI, Eli Lilly, AstraZeneca, Abbvie, Servier, Mylan, Gilead, Amgen; he was the scientific director of a Master's programme on pharmacovigilance, pharmacoepidemiology and real-world evidence which has received non-conditional grants from various pharmaceutical companies; he coordinated a pharmacoepidemiology team at the University of Messina until Oct 2020, which has received funding for conducting observational studies from various pharmaceutical companies (Boehringer Ingelheim, Daichii Sankyo, PTC Pharmaceuticals). He is also scientific coordinator of the academic spin-off 'INSPIRE srl' which has received funding for conducting observational studies from contract research organisations (RTI Health Solutions, Pharmo Institute N.V.). All the above-mentioned activities are not related to the topic of the manuscript. Carmen Ferrajolo declares that she is a member and vice-chief of academic spin-off 'INSPIRE SRL- INnovative Solutions for medical Prediction and big data Integration in REal world setting SRL', which has received funding for conducting observational studies from various pharmaceutical companies. The other authors have no conflicts of interest to disclose.

Ethics approval Not applicable.

Consent to participate Not applicable.

Consent for publication Not applicable.

Availability of data and material Not applicable.

Code availability Not applicable.
Author contributions All authors contributed equally to this work. All authors read and approved the final version.

\section{References}

1. Polack FP, Thomas SJ, Kitchin N, et al. Safety and efficacy of the BNT162b2 mRNA Covid-19 vaccine. N Engl J Med. 2020;383(27):2603-15. https://doi.org/10.1056/NEJMoa2034 577.

2. Baden LR, El Sahly HM, Essink B, et al. Efficacy and safety of the mRNA-1273 SARS-CoV-2 vaccine. N Engl J Med. 2021;384(5):403-16. https://doi.org/10.1056/NEJMoa2035389.

3. Sadoff J, Gray G, Vandebosch A, et al. Safety and efficacy of single-dose Ad26.COV2.S vaccine against Covid-19. N Engl J Med. 2021;384(23):2187-201. https://doi.org/10.1056/NEJMo a2101544.

4. Voysey M, Clemens SAC, Madhi SA, et al. Safety and efficacy of the ChAdOx1 nCoV-19 vaccine (AZD1222) against SARSCoV-2: an interim analysis of four randomised controlled trials in Brazil, South Africa, and the UK. Lancet. 2021;397(10269):99111. https://doi.org/10.1016/S0140-6736(20)32661-1.

5. Viner RM, Mytton OT, Bonell C, et al. Susceptibility to SARSCoV-2 infection among children and adolescents compared with adults: a systematic review and meta-analysis. JAMA Pediatr. 2021;175(2):212.

6. Lavezzo E, Franchin E, Ciavarella C, et al. Suppression of a SARS-CoV-2 outbreak in the Italian municipality of Vo'. Nature. 2021;590(7844):E11. https://doi.org/10.1038/ s41586-020-2956-7.

7. COVID-19 National Emergency Response Center, Epidemiology and Case Management Team KC for DC and P. Coronavirus Disease-19: the first 7,755 cases in the Republic of Korea. Osong public Heal Res Perspect. 2020;11(2):85-90. https://doi.org/10. 24171/j.phrp.2020.11.2.05.

8. Gudbjartsson DF, Helgason A, Jonsson H, et al. Spread of SARS-CoV-2 in the Icelandic population. N Engl J Med. 2020;382(24):2302-15. https://doi.org/10.1056/NEJMoa2006 100.

9. Fantini MP, Reno C, Biserni GB, Savoia E, Lanari M. COVID-19 and the re-opening of schools: a policy maker's dilemma. Ital J Pediatr. 2020;46(1):79. https://doi.org/10.1186/ s13052-020-00844-1.

10. Istituto Nazionale di Statistica (ISTAT). PRIMI RISULTATI DELL'INDAGINE DI SIEROPREVALENZA SUL SARSCOV-2. 2020. https://www.istat.it/it/archivio/246156. Accessed 6 Aug 2021.

11. Li Q, Guan X, Wu P, et al. Early transmission dynamics in Wuhan, China, of novel coronavirus-infected pneumonia. N Engl J Med. 2020;382(13):1199-207. https://doi.org/10.1056/NEJMo a2001316.

12. European Centre for Disease Prevention and Control (ECDC). COVID-19 in children and the role of school settings in COVID19 transmission. 2020. https://www.ecdc.europa.eu/sites/defau lt/files/documents/COVID-19-schools-transmission-August\% 202020.pdf. Accessed 6 Aug 2021.

13. JCVI statement on COVID-19 vaccination of children and young people aged 12 to 17 years: 4 August 2021. 2021. https://www. gov.uk/government/publications/jcvi-statement-august-2021covid-19-vaccination-of-children-and-young-people-aged-12to-17-years/jcvi-statement-on-covid-19-vaccination-of-childrenand-young-people-aged-12-to-17-years-4-august-2021. Accessed 6 Aug 2021. 
14. Willeit P, Krause R, Lamprecht B, et al. Prevalence of RT-qPCRdetected SARS-CoV-2 infection at schools: first results from the Austrian School-SARS-CoV-2 prospective cohort study. Lancet Reg Heal Europe. 2021. https://doi.org/10.1016/j.lanepe.2021. 100086.

15. Dong Y, Mo X, Hu Y, et al. Epidemiology of COVID-19 among children in China. Pediatrics. 2020;145(6): e20200702. https:// doi.org/10.1542/peds.2020-0702.

16. Hong H, Wang Y, Chung H-T, Chen C-J. Clinical characteristics of novel coronavirus disease 2019 (COVID-19) in newborns, infants and children. Pediatr Neonatol. 2020;61(2):131-2. https:// doi.org/10.1016/j.pedneo.2020.03.001.

17. Mehta NS, Mytton OT, Mullins EWS, et al. SARS-CoV-2 (COVID-19): what do we know about children? A systematic review. Clin Infect Dis. 2020;71(9):2469-79. https://doi.org/10. 1093/cid/ciaa556.

18. Centers for Disease Control and Prevention (CDC). COVIDNET. 2021. https://gis.cdc.gov/grasp/covidnet/covid19_3.html. Accessed 6 Aug 2021.

19. Shekerdemian LS, Mahmood NR, Wolfe KK, et al. Characteristics and outcomes of children with coronavirus disease 2019 (COVID-19) infection admitted to US and Canadian pediatric intensive care units. JAMA Pediatr. 2020;174(9):868-73. https:// doi.org/10.1001/jamapediatrics.2020.1948.

20. Ludvigsson JF. Systematic review of COVID-19 in children shows milder cases and a better prognosis than adults. Acta Paediatr. 2020;109(6):1088-95. https://doi.org/10.1111/apa.15270.

21. World Health Organization. Multisystem inflammatory syndrome in children and adolescents temporally related to COVID-19. 2020. https://www.who.int/news-room/commentaries/detail/ multisystem-inflammatory-syndrome-in-children-and-adole scents-with-covid-19. Accessed 6 Aug 2021.

22. Park YJ, Choe YJ, Park O, et al. Contact tracing during coronavirus disease outbreak, South Korea, 2020. Emerg Infect Dis. 2020;26(10):2465-8. https://doi.org/10.3201/eid2610.201315.

23. Bellino S, Rota MC, Riccardo F, et al. Pediatric COVID-19 cases prelockdown and postlockdown in Italy. Pediatrics. 2021;147(2): e2020035238. https://doi.org/10.1542/peds.2020-035238.

24. European Centre for Disease Prevention and Control (ECDC). COVID-19 in children and the role of school settings in transmission—second update. 2021. https://www.ecdc.europa.eu/en/publi cations-data/children-and-school-settings-covid-19-transmission. Accessed 6 Aug 2021.

25. Brookman S, Cook J, Zucherman M, Broughton S, Harman K, Gupta A. Effect of the new SARS-CoV-2 variant B.1.1.7 on children and young people. Lancet Child Adolesc Heal. 2021;5(4):e9-10. https://doi.org/10.1016/S2352-4642(21) 00030-4.

26. Frenck RW, Klein NP, Kitchin N, Gurtman A, Absalon J, Lockhart S, et al. Safety, immunogenicity, and efficacy of the BNT162b2 Covid-19 vaccine in adolescents. N Engl J Med. 2021;385(3):239-50. https://doi.org/10.1056/NEJMoa2107456.

27. European Medicines Agency. COVID-19 vaccine Spikevax approved for children aged 12 to 17 in EU. 2021. https://www. ema.europa.eu/en/news/covid-19-vaccine-spikevax-approvedchildren-aged-12-17-eu. Accessed 6 Aug 2021.

28. COVID19 vaccine tracker. Approved vaccines. 2021. https:// covid19.trackvaccines.org/vaccines/approved/. Accessed 6 Aug 2021.

29. Han B, Song Y, Li C, et al. Safety, tolerability, and immunogenicity of an inactivated SARS-CoV-2 vaccine (CoronaVac) in healthy children and adolescents: a double-blind, randomised, controlled, phase 1/2 clinical trial. Lancet Infect Dis. 2021;S1473-3099(21):00319-24. https://doi.org/10.1016/S14733099(21)00319-4.
30. Black SB, Law B, Chen RT, et al. The critical role of background rates of possible adverse events in the assessment of COVID-19 vaccine safety. Vaccine. 2021;39(19):2712-8. https://doi.org/10. 1016/j.vaccine.2021.03.016.

31. Gubernot D, Jazwa A, Niu M, et al. U.S. Population-Based background incidence rates of medical conditions for use in safety assessment of COVID-19 vaccines. Vaccine. 2021;39(28):366677. https://doi.org/10.1016/j.vaccine.2021.05.016.

32. European Medicines Agency. EMA commissions independent research to prepare for real-world monitoring of COVID-19 vaccines. 2020. https://www.ema.europa.eu/en/news/ema-commi ssions-independent-research-prepare-real-world-monitoringcovid-19-vaccines. Accessed 6 Aug 2021.

33. Gargano JW, Wallace M, Hadler SC, et al. Use of mRNA COVID-19 vaccine after reports of myocarditis among vaccine recipients: update from the advisory committee on immunization practices-United States, June 2021. MMWR Morb Mortal Wkly Rep. 2021;70(27):977-82. https://doi.org/10.15585/mmwr. mm7027e2.

34. Bozkurt B, Kamat I, Hotez PJ. Myocarditis with COVID-19 mRNA vaccines. Circulation. 2021. https://doi.org/10.1161/ CIRCULATIONAHA.121.056135.

35. European Medicines Agency. First COVID-19 vaccine approved for children aged 12 to 15 in EU. 2021. https://www.ema.europa. eu/en/news/first-covid-19-vaccine-approved-children-aged-1215-eu. Accessed 6 Aug 2021.

36. Wilkinson D, Finlay I, Pollard AJ, Forsberg L, Skelton A. Should we delay covid-19 vaccination in children? BMJ. 2021;8(374): n1687. https://doi.org/10.1136/bmj.n1687.

37. Lavine JS, Bjornstad O, Antia R. Vaccinating children against SARS-CoV-2. BMJ. 2021;13(373): n1197. https://doi.org/10. 1136/bmj.n1197.

38. Feldstein LR, Tenforde MW, Friedman KG, et al. Characteristics and outcomes of US children and adolescents with multisystem inflammatory syndrome in children (MIS-C) compared with severe acute COVID-19. JAMA. 2021;325(11):1074-87. https:// doi.org/10.1001/jama.2021.2091.

39. Levin M. Childhood multisystem inflammatory syndrome-a new challenge in the pandemic. N Engl J Med. 2020;383(4):3935. https://doi.org/10.1056/NEJMe2023158.

40. Godfred-Cato S, Bryant B, Leung J, et al. COVID-19-associated multisystem inflammatory syndrome in children-United States, March-July 2020. MMWR Morb Mortal Wkly Rep. 2020;69(32):1074-80. https://doi.org/10.15585/mmwr.mm693 $2 \mathrm{e} 2$.

41. Toubiana J, Poirault C, Corsia A, Bajolle F, Fourgeaud J, Angoulvant $\mathrm{F}$, et al. Kawasaki-like multisystem inflammatory syndrome in children during the covid-19 pandemic in Paris, France: prospective observational study. BMJ. 2020;3(369): m2094. https:// doi.org/10.1136/bmj.m2094.

42. Centers for Disease Control and Prevention (CDC). COVID-19 Vaccines for Children and Teens. 2021. https://www.cdc.gov/ coronavirus/2019-ncov/vaccines/recommendations/adolescents. html. Accessed 6 Aug 2021.

43. European Centre for Disease Prevention and Control (ECDC). ECDC report outlines considerations for COVID-19 vaccination of adolescents. 2021. https://www.ecdc.europa.eu/en/newsevents/ecdc-report-outlines-considerations-covid-19-vacci nation-adolescents.

44. Morgan L, Schwartz JL, Sisti DA. COVID-19 vaccination of minors without parental consent. JAMA Pediatr. 2021. https:// doi.org/10.1001/jamapediatrics.2021.1855.

45. Allotey J, Stallings E, Bonet M, et al. Clinical manifestations, risk factors, and maternal and perinatal outcomes of coronavirus disease 2019 in pregnancy: living systematic review and 
meta-analysis. BMJ. 2020;1(370): m3320. https://doi.org/10. 1136/bmj.m3320.

46. World Health Organization. Coronavirus disease (COVID-19): Pregnancy and childbirth. 2020. https://www.who.int/newsroom/q-a-detail/coronavirus-disease-covid-19-pregnancy-andchildbirth. Accessed 6 Aug 2021.

47. Centers for Disease Control and Prevention (CDC). Data on COVID-19 during Pregnancy: Severity of Maternal Illness. 2021. https://stacks.cdc.gov/view/cdc/99912. Accessed 6 Aug 2021.

48. Zambrano LD, Ellington S, Strid P, et al. Update: characteristics of symptomatic women of reproductive age with laboratoryconfirmed SARS-CoV-2 Infection by Pregnancy Status-United States, January 22-October 3, 2020. MMWR Morb Mortal Wkly Rep. 2020;69(44):1641-7. https://doi.org/10.15585/mmwr. $\mathrm{mm} 6944 \mathrm{e} 3$

49. Richtmann R, Torloni MR, Oyamada Otani AR, et al. Fetal deaths in pregnancies with SARS-CoV-2 infection in Brazil: a case series. Case Rep Women's Heal. 2020;12(27): e00243. https://doi.org/10.1016/j.crwh.2020.e00243.

50. Gurol-Urganci I, Jardine JE, Carroll F, et al. Maternal and perinatal outcomes of pregnant women with SARS-CoV-2 infection at the time of birth in England: national cohort study. Am J Obstet Gynecol. 2021;S0002-9378(21):00565-72. https://doi.org/10. 1016/j.ajog.2021.05.016.

51. Linehan L, O'Donoghue K, Dineen S, White J, Higgins JR, Fitzgerald B. SARS-CoV-2 placentitis: an uncommon complication of maternal COVID-19. Placenta. 2021;15(104):261-6. https://doi.org/10.1016/j.placenta.2021.01.012.

52. Baral G, Shrestha O, Baral RS. Thrombotic pathology in placenta of COVID positive pregnancy. J Nepal Health Res Counc. 2021;19(1):206-8. https://doi.org/10.33314/jnhrc.v19i1.3403.

53. Wei SQ, Bilodeau-Bertrand M, Liu S, Auger N. The impact of COVID-19 on pregnancy outcomes: a systematic review and meta-analysis. Can Med Assoc J. 2021;193(16):E540-8. https:// doi.org/10.1503/cmaj.202604.

54. Ravaldi C, Wilson A, Ricca V, Homer C, Vannacci A. Pregnant women voice their concerns and birth expectations during the COVID-19 pandemic in Italy. Women Birth. 2021;34(4):335-43. https://doi.org/10.1016/j.wombi.2020.07.002.

55. Ravaldi C, Ricca V, Wilson A, Homer C, Vannacci A. Previous psychopathology predicted severe COVID-19 concern, anxiety, and PTSD symptoms in pregnant women during "lockdown" in Italy. Arch Womens Ment Health. 2020;23(6):783-6. https://doi. org/10.1007/s00737-020-01086-0.

56. Centers for Disease Control and Prevention (CDC). COVID-19 Vaccines While Pregnant or Breastfeeding. 2021. https://www. cdc.gov/coronavirus/2019-ncov/vaccines/recommendations/ pregnancy.html. Accessed 6 Aug 2021.

57. Goldshtein I, Nevo D, Steinberg DM, et al. Association between BNT162b2 vaccination and incidence of SARS-CoV-2 infection in pregnant women. JAMA. 2021;12: e2111035. https://doi.org/ 10.1001/jama.2021.11035.

58. Prabhu M, Murphy EA, Sukhu AC, et al. Antibody response to coronavirus disease 2019 (COVID-19) messenger RNA vaccination in pregnant women and transplacental passage into cord blood. Obstet Gynecol. 2021;138(2):278-80. https://doi.org/10. 1097/AOG.0000000000004438.

59. Gray KJ, Bordt EA, Atyeo C, et al. Coronavirus disease 2019 vaccine response in pregnant and lactating women: a cohort study. Am J Obstet Gynecol. 2021;S0002-9378(21):00187-93. https://doi.org/10.1016/j.ajog.2021.03.023.

60. Perl SH, Uzan-Yulzari A, Klainer H, Asiskovich L, Youngster M, Rinott E, et al. SARS-CoV-2-specific antibodies in breast milk after COVID-19 vaccination of breastfeeding women. JAMA. 2021;325(19):2013-4. https://doi.org/10.1001/jama.2021.5782.
61. Centers for Disease Control and Prevention (CDC). V-safe COVID-19 Vaccine Pregnancy Registry. 2021. https://www.cdc. gov/coronavirus/2019-ncov/vaccines/safety/vsafepregnancyregist ry.html. Accessed 6 Aug 2021.

62. Shimabukuro TT, Kim SY, Myers TR, et al. Preliminary findings of mRNA Covid-19 vaccine safety in pregnant persons. N Engl J Med. 2021;384(24):2273-82. https://doi.org/10.1056/NEJMo a2104983.

63. American College of Obstetricians and Gynecologists' Committee on Practice Bulletins-Gynecology. ACOG Practice Bulletin No. 200: early pregnancy loss. Obstet Gynecol. 2018;132(5):e197-207. https://doi.org/10.1097/AOG.00000 00000002899.

64. The American College of Obstetricians and Gynecologists. COVID-19 Vaccination Considerations for Obstetric-Gynecologic Care. 2021. https://www.acog.org/clinical/clinical-guida nce/practice-advisory/articles/2020/12/covid-19-vaccinationconsiderations-for-obstetric-gynecologic-care. Accessed 6 Aug 2021.

65. Royal College of Obstetricians \& Gynaecologists. COVID-19 vaccines, pregnancy and breastfeeding. 2021. https://www.rcog. org.uk/en/guidelines-research-services/coronavirus-covid-19pregnancy-and-womens-health/covid-19-vaccines-and-pregn ancy/covid-19-vaccines-pregnancy-and-breastfeeding/. Accessed 6 Aug 2021.

66. The Royal Australian and New Zeland College of Obstetricians and Gynaecologists. Pregnant women are now eligible for the Pfizer COVID-19 vaccine. 2021. https://ranzcog.edu.au/news/ pregnant-women-are-now-eligible-for-the-pfizer-cov. Accessed 6 Aug 2021.

67. Società Italiana di Ginecologia e Ostetricia. Gravidanza e Vaccinazione anti COVID. 2021. https://www.sigo.it/wp-content/ uploads/2021/05/PositionPaper_Gravidanza_Vaccinazione_anti_ COVID_05.05.2021.pdf. Accessed 6 Aug 2021.

68. World Health Organization. Update on WHO Interim recommendations on COVID-19 vaccination of pregnant and lactating women. 2021. https://www.who.int/publications/m/item/updateon-who-interim-recommendations-on-covid-19-vaccination-ofpregnant-and-lactating-women. Accessed 6 Aug 2021.

69. Chavan M, Qureshi H, Karnati S, Kollikonda S. COVID-19 vaccination in pregnancy: the benefits outweigh the risks. J Obstet Gynaecol Canada. 2021;43(7):814-6. https://doi.org/10.1016/j. jogc.2021.03.010.

70. Kuderer NM, Choueiri TK, Shah DP, et al. Clinical impact of COVID-19 on patients with cancer (CCC19): a cohort study. Lancet. 2020;395(10241):1907-18. https://doi.org/10.1016/ S0140-6736(20)31187-9.

71. Lee LY, Cazier J-B, Angelis V, et al. COVID-19 mortality in patients with cancer on chemotherapy or other anticancer treatments: a prospective cohort study. Lancet. 2020;395(10241):1919-26. https://doi.org/10.1016/S01406736(20)31173-9.

72. He W, Chen L, Chen L, et al. COVID-19 in persons with haematological cancers. Leukemia. 2020;34(6):1637-45. https://doi. org/10.1038/s41375-020-0836-7.

73. Martín-Moro F, Marquet J, Piris M, et al. Survival study of hospitalised patients with concurrent COVID-19 and haematological malignancies. Br J Haematol. 2020;190(1):e16-20. https://doi. org/10.1111/bjh.16801.

74. Malard F, Genthon A, Brissot E, et al. COVID-19 outcomes in patients with hematologic disease. Bone Marrow Transplant. 2020;55(11):2180-4. https://doi.org/10.1038/ s41409-020-0931-4.

75. Aries JA, Davies JK, Auer RL, et al. Clinical outcome of coronavirus disease 2019 in haemato-oncology patients. Br J Haematol. 2020;190(2):e64-7. https://doi.org/10.1111/bjh.16852. 
76. Kates OS, Haydel BM, Florman SS, et al. COVID-19 in solid organ transplant: a multi-center cohort study. Clin Infect Dis. 2020. https://doi.org/10.1093/cid/ciaa1097.

77. Sharma A, Bhatt NS, St Martin A, et al. Clinical characteristics and outcomes of COVID-19 in haematopoietic stem-cell transplantation recipients: an observational cohort study. Lancet Haematol. 2021;8(3):e185-93. https://doi.org/10.1016/S23523026(20)30429-4.

78. Fung M, Babik JM. COVID-19 in immunocompromised hosts: what we know so far. Clin Infect Dis. 2021;72(2):340-50. https:// doi.org/10.1093/cid/ciaa863.

79. Tesoriero JM, Swain C-AE, Pierce JL, et al. COVID-19 outcomes among persons living with or without diagnosed HIV infection in New York State. JAMA Netw open. 2021;4(2):e2037069. https:// doi.org/10.1001/jamanetworkopen.2020.37069.

80. Akiyama S, Hamdeh S, Micic D, Sakuraba A. Prevalence and clinical outcomes of COVID-19 in patients with autoimmune diseases: a systematic review and meta-analysis. Ann Rheum Dis. 2020. https://doi.org/10.1136/annrheumdis-2020-218946.

81. D'Silva KM, Wallace ZS. COVID-19 and rheumatoid arthritis. Curr Opin Rheumatol. 2021;33(3):255-61. https://doi.org/10. 1097/BOR.0000000000000786.

82. Spila Alegiani S, Crisafulli S, Giorgi Rossi P, et al. Risk of coronavirus disease 2019 hospitalization and mortality in rheumatic patients treated with hydroxychloroquine or other conventional disease-modifying anti-rheumatic drugs in Italy. Rheumatology. 2021. https://doi.org/10.1093/rheumatology/keab348.

83. Jee J, Foote MB, Lumish M, et al. Chemotherapy and COVID-19 outcomes in patients with cancer. J Clin Oncol. 2020;38(30):3538-46. https://doi.org/10.1200/JCO.20.01307.

84. Grivas P, Khaki AR, Wise-Draper TM, et al. Association of clinical factors and recent anticancer therapy with COVID-19 severity among patients with cancer: a report from the COVID-19 and Cancer Consortium. Ann Oncol Off J Eur Soc Med Oncol. 2021;32(6):787-800. https://doi.org/10.1016/j.annonc.2021.02. 024.

85. Boyarsky BJ, Werbel WA, Avery RK, et al. Antibody response to 2-Dose SARS-CoV-2 mRNA vaccine series in solid organ transplant recipients. JAMA. 2021;325(21):2204-6. https://doi. org/10.1001/jama.2021.7489.

86. Shrotri M, Fragaszy E, Geismar C, et al. Spike-antibody responses to ChAdOx1 and BNT162b2 vaccines by demographic and clinical factors (Virus Watch study). medRxiv. 2021. https:// doi.org/10.1101/2021.05.12.21257102.

87. Benotmane I, Gautier-Vargas G, Cognard N, et al. Weak antiSARS-CoV-2 antibody response after the first injection of an mRNA COVID-19 vaccine in kidney transplant recipients. Kidney Int. 2021;99(6):1487-9. https://doi.org/10.1016/j.kint.2021. 03.014.

88. Yi SG, Knight RJ, Graviss EA, et al. Kidney transplant recipients rarely show an early antibody response following the first COVID-19 vaccine administration. Transplantation. 2021;105(7):e72-3. https://doi.org/10.1097/TP.0000000000 003764 .

89. Boyarsky BJ, Ruddy JA, Connolly CM, et al. Antibody response to a single dose of SARS-CoV-2 mRNA vaccine in patients with rheumatic and musculoskeletal diseases. Ann Rheum Dis. 2021. https://doi.org/10.1136/annrheumdis-2021-220289.

90. Palich R, Veyri M, Marot S, Vozy A, Gligorov J, Maingon $\mathrm{P}$, et al. Weak immunogenicity after a single dose of SARSCoV-2 mRNA vaccine in treated cancer patients. Ann Oncol. 2021;32(8):1051-3. https://doi.org/10.1016/j.annonc.2021.04. 020 .

91. Agha M, Blake M, Chilleo C, Wells A, Haidar G. Suboptimal response to COVID-19 mRNA vaccines in hematologic malignancies patients. medRxiv [Preprint]. 2021. https://doi. org/10.1101/2021.04.06.21254949.

92. Monin L, Laing AG, Muñoz-Ruiz M, et al. Safety and immunogenicity of one versus two doses of the COVID-19 vaccine BNT162b2 for patients with cancer: interim analysis of a prospective observational study. Lancet Oncol. 2021;22(6):765-78. https://doi.org/10.1016/S1470-2045(21)00213-8.

93. Peled Y, Ram E, Lavee J, et al. BNT162b2 vaccination in heart transplant recipients: clinical experience and antibody response. J Hear Lung Transplant. 2021;40(8):759-62. https://doi.org/10. 1016/j.healun.2021.04.003.

94. Rincon-Arevalo H, Choi M, Stefanski A-L, et al. Impaired humoral immunity to SARS-CoV-2 BNT162b2 vaccine in kidney transplant recipients and dialysis patients. Sci Immunol. 2021;6(60):eabj1031. https://doi.org/10.1126/sciimmunol.abj10 31.

95. Havlin J, Svorcova M, Dvorackova E, et al. Immunogenicity of BNT162b2 mRNA COVID-19 vaccine and SARS-CoV-2 infection in lung transplant recipients. J Heart Lung Transplant. 2021;40(8):754-8. https://doi.org/10.1016/j.healun.2021.05.004.

96. Danthu C, Hantz S, Dahlem A, et al. Humoral response after SARS-Cov-2 mRNA vaccine in a cohort of hemodialysis patients and kidney transplant recipients. J Am Soc Nephrol. 2021. https://doi.org/10.1681/ASN.2021040490.

97. European Medicines Agency. COVID-19 Vaccine Janssen. 2021. https://www.ema.europa.eu/en/medicines/human/EPAR/covid19-vaccine-janssen. Accessed 6 Aug 2021.

98. European Medicines Agency. Comirnaty. 2021. https://www.ema. europa.eu/en/medicines/human/EPAR/comirnaty. Accessed 6 Aug 2021.

99. European Medicines Agency. Spikevax (previously COVID-19 Vaccine Moderna). 2021. https://www.ema.europa.eu/en/medic ines/human/EPAR/spikevax-previously-covid-19-vaccine-moder na. Accessed 6 Aug 2021.

100. European Medicines Agency. Vaxzevria (previously COVID-19 Vaccine AstraZeneca). 2021. https://www.ema.europa.eu/en/ medicines/human/EPAR/vaxzevria-previously-covid-19-vacci ne-astrazeneca. Accessed 6 Aug 2021.

101. Shroff RT, Chalasani P, Wei R, et al. Immune responses to COVID-19 mRNA vaccines in patients with solid tumors on active, immunosuppressive cancer therapy. medRxiv. 2021. https://doi.org/10.1101/2021.05.13.21257129.

102. Kamar N, Abravanel F, Marion O, Couat C, Izopet J, Del Bello A. Three doses of an mRNA Covid-19 vaccine in solid-organ transplant recipients. N Engl J Med. 2021. https://doi.org/10. 1056/NEJMc2108861

103. Whitaker HJ, Tsang RS, Byford R, et al. Pfizer-BioNTech and Oxford AstraZeneca COVID-19 vaccine effectiveness and immune response among individuals in clinical risk groups. pre-print. 2021. https://khub.net/documents/135939561/43098 6542/RCGP+VE+riskgroups+paper.pdf/a6b54cd9-419d-9b63e2bf-5dc796f5a91f. Accessed 6 Aug 2021.

104. Sonani B, Aslam F, Goyal A, Patel J, Bansal P. COVID-19 vaccination in immunocompromised patients. Clin Rheumatol. 2021;40(2):797-8. https://doi.org/10.1007/s10067-020-05547-w.

105. Curtis JR, Johnson SR, Anthony DD, et al. American College of Rheumatology Guidance for COVID-19 vaccination in patients with rheumatic and musculoskeletal diseases: version 1 . Arthritis Rheumatol. 2021;73(7):1093-107. https://doi.org/10.1002/art. 41734.

106. Daniel Kaul. How Should We Advise Our Immunocompromised Patients After COVID-19 Vaccination? NEJM J. Watch. 2021. https://www.jwatch.org/na53599/2021/06/14/how-should-weadvise-our-immunocompromised-patients-after. Accessed 6 Aug 2021. 
107. Park JK, Lee EB, Shin K, et al. COVID-19 vaccination in patients with autoimmune inflammatory rheumatic diseases: clinical guidance of the Korean College of Rheumatology. J Korean Med Sci. 2021;36(12): e95. https://doi.org/10.3346/jkms.2021.36.e95.

108. Pawankar R. Allergic diseases and asthma: a global public health concern and a call to action. World Allergy Organ J. 2014;7(1):12. https://doi.org/10.1186/1939-4551-7-12.

109. Pawankar R, Canonica GW, Holgate ST, Lockey RF, Blaiss MS. White Book on Allergy. World Allergy Organ. 2011. http:// www.worldallergy.org/UserFiles/file/WhiteBook2-2013-v8.pdf. Accessed 6 Aug 2021.

110. World Health Organization. Asthma. 2021. https://www.who.int/ news-room/fact-sheets/detail/asthma. Accessed 6 Aug 2021.

111. Shi L, Xu J, Xiao W, et al. Asthma in patients with coronavirus disease 2019: a systematic review and meta-analysis. Ann Allergy Asthma Immunol. 2021;126(5):524-34. https://doi.org/ 10.1016/j.anai.2021.02.013.

112. Yang JM, Koh HY, Moon SY, et al. Allergic disorders and susceptibility to and severity of COVID-19: a nationwide cohort study. J Allergy Clin Immunol. 2020;146(4):790-8. https://doi. org/10.1016/j.jaci.2020.08.008.

113. Sampath V, Rabinowitz G, Shah M, et al. Vaccines and allergic reactions: the past, the current COVID-19 pandemic, and future perspectives. Allergy. 2021;76(6):1640-60. https://doi.org/10. 1111/all.14840.

114. CDC COVID-19 Response Team; Food and Drug Administration. Allergic reactions including anaphylaxis after receipt of the first dose of Pfizer-BioNTech COVID-19 vaccine-United States, December 14-23, 2020. MMWR Morb Mortal Wkly Rep. 2021;70(2):46-51. https://doi.org/10.15585/mmwr.mm7002e1.

115. CDC COVID-19 Response Team; Food and Drug Administration. Allergic reactions including anaphylaxis after receipt of the first dose of Moderna COVID-19 Vaccine-United States, December 21, 2020-January 10, 2021. MMWR Morb Mortal Wkly Rep. 2021;70(4):125-9. https://doi.org/10.15585/mmwr. mm7004e1.

116. Shimabukuro TT, Cole M, Su JR. Reports of anaphylaxis after receipt of mRNA COVID-19 vaccines in the US-December 14, 2020-January 18, 2021. JAMA. 2021;325(11):1101-2. https:// doi.org/10.1001/jama.2021.1967.

117. Blumenthal KG, Robinson LB, Camargo CA, Shenoy ES, Banerji A, Landman AB, et al. Acute allergic reactions to mRNA COVID-19 vaccines. JAMA. 2021;325(15):1562-5. https://doi. org/10.1001/jama.2021.3976.

118. Krantz MS, Kwah JH, Stone CA, et al. Safety evaluation of the second dose of messenger RNA COVID-19 vaccines in patients with immediate reactions to the first dose. JAMA Intern Med [Internet]. 2021. https://doi.org/10.1001/jamainternmed.2021. 3779.

119. BSACI. Update on MHRA decision re: Pfizer COVID-19 Vaccination 30.12.20. 2020. https://www.bsaci.org/update-on-mhradecision-re-pfizer-covid-19-vaccination/. Accessed 6 Aug 2021.

120. Erdeljic TV. Anaphylaxis associated with the mRNA COVID19 vaccines: approach to allergy investigation. Clin Immunol. 2021;227: 108748. https://doi.org/10.1016/j.clim.2021.108748.

121. Stone CA, Liu Y, Relling MV, et al. Immediate hypersensitivity to polyethylene glycols and polysorbates: more common than we have recognized. J Allergy Clin Immunol Pract. 2019;7(5):15331540.e8. https://doi.org/10.1016/j.jaip.2018.12.003.

122. Sellaturay P, Nasser S, Ewan P. Polyethylene glycol-induced systemic allergic reactions (anaphylaxis). J allergy Clin Immunol Pract. 2021;9(2):670-5. https://doi.org/10.1016/j.jaip.2020.09. 029.

123. Centers for Disease Control and Prevention (CDC). COVID-19 Vaccines for People with Allergies. 2021. https://www.cdc.gov/ coronavirus/2019-ncov/vaccines/recommendations/specificgroups/allergies.html. Accessed 6 Aug 2021.

124. Sokolowska M, Eiwegger T, Ollert M, Torres MJ, Barber D, Del Giacco S, et al. EAACI statement on the diagnosis, management and prevention of severe allergic reactions to COVID-19 vaccines. Allergy. 2021;76(6):1629-39. https://doi.org/10.1111/all. 14739.

125. American College of Allergy, Asthma \& Immunology. ACAAI Updates to Guidance on Risk of Allergic Reactions to COVID19 Vaccines. 2021. https://acaai.org/news/acaai-updates-guida nce-risk-allergic-reactions-covid-19-vaccines. Accessed 6 Aug 2021.

126. COVID-19: the green book, chapter 14a. 2021. https://www.gov. uk/government/publications/covid-19-the-green-book-chapter14a. Accessed 6 Aug 2021.

127. Altrichter S, Wöhrl S, Horak F, et al. Answers to burning questions for clinical allergologists related to the new COVID-19 vaccines. Allergo J Int. 2021;14:1-7. https://doi.org/10.1007/ s40629-021-00177-3.

128. AIFA. FAQ - COVID-19 vaccines. 2021. https://www.aifa.gov. it/en/domande-e-risposte-su-vaccini-covid-19. Accessed 6 Aug 2021.

129. Greenhawt M, Abrams EM, Shaker M, et al. The risk of allergic reaction to SARS-CoV-2 vaccines and recommended evaluation and management: a systematic review, meta-analysis, GRADE assessment, and international consensus approach. J Allergy Clin Immunol Pract. 2021;S2213-2198(21):00671-81. https://doi.org/ 10.1016/j.jaip.2021.06.006.

130. Hansen CH, Michlmayr D, Gubbels SM, Mølbak K, Ethelberg S. Assessment of protection against reinfection with SARS-CoV-2 among 4 million PCR-tested individuals in Denmark in 2020: a population-level observational study. Lancet. 2021;397(10280):1204-12. https://doi.org/10.1016/S01406736(21)00575-4.

131. Wang Z, Muecksch F, Schaefer-Babajew D, et al. Naturally enhanced neutralizing breadth against SARS-CoV-2 one year after infection. Nature. 2021;595(7867):426-31. https://doi.org/ 10.1038/s41586-021-03696-9.

132. Camara C, Lozano-Ojalvo D, Lopez-Granados E, et al. Differential effects of the second SARS-CoV-2 mRNA vaccine dose on $\mathrm{T}$ cell immunity in naïve and COVID-19 recovered individuals. bioRxiv. 2021. https://doi.org/10.1101/2021.03.22.436441.

133. Mazzoni A, Di Lauria N, Maggi L, et al. First-dose mRNA vaccination is sufficient to reactivate immunological memory to SARS-CoV-2 in subjects who have recovered from COVID-19. J Clin Invest. 2021;131(12): e149150. https://doi.org/10.1172/ JCI149150.

134. Nadesalingam A, Cantoni D, Wells DA, et al. Paucity and discordance of neutralising antibody responses to SARS-CoV-2 VOCs in vaccinated immunodeficient patients and health-care workers in the UK. Lancet Microbe. 2021. https://doi.org/10. 1016/S2666-5247(21)00157-9.

135. Sasikala M, Shashidhar J, Deepika G, et al. Immunological memory and neutralizing activity to a single dose of COVID19 vaccine in previously infected individuals. Int J Infect Dis. 2021;108:183-6. https://doi.org/10.1016/j.ijid.2021.05.034.

136. Shrestha NK, Burke PC, Nowacki AS, Terpeluk P, Gordon ST. Necessity of COVID-19 vaccination in previously infected individuals. medRxiv. 2021. https://doi.org/10.1101/2021.06.01. 21258176.

137. Alhazmi A, Alamer E, Daws D, et al. Evaluation of side effects associated with COVID-19 vaccines in Saudi Arabia. Vaccines. 2021;9(6):674. https://doi.org/10.3390/vaccines9060674.

138. Dolgin E. Is one vaccine dose enough if you've had COVID? What the science says. Nature. 2021;595(7866):161-2. https:// doi.org/10.1038/d41586-021-01609-4. 
139. Lurie N, Saville M, Hatchett R, Halton J. Developing covid-19 vaccines at pandemic speed. N Engl J Med. 2020;382(21):196973. https://doi.org/10.1056/NEJMp2005630.

140. Montalti M, Soldà G, Di Valerio Z, et al. ROCCA observational study: early results on safety of Sputnik V vaccine (GamCOVID-Vac) in the Republic of San Marino using active surveillance. EClin Med. 2021;8(38): 101027. https://doi.org/10.1016/j. eclinm.2021.101027.

141. Menni C, Klaser K, May A, et al. Vaccine side-effects and SARS-CoV-2 infection after vaccination in users of the COVID
Symptom Study app in the UK: a prospective observational study. Lancet Infect Dis. 2021;21(7):939-49. https://doi.org/10. 1016/S1473-3099(21)00224-3.

142. ORCHESTRA project. https://orchestra-cohort.eu. Accessed 6 Aug 2021.

143. European Medicines Agency. Monitoring of COVID-19 medicines. 2021. https://www.ema.europa.eu/en/human-regulatory/ overview/public-health-threats/coronavirus-disease-covid-19/ treatments-vaccines/monitoring-covid-19-medicines-0. Accessed 6 Aug 2021.

\section{Authors and Affiliations}

\section{Nicoletta Luxi ${ }^{1}$ - Alexia Giovanazzi ${ }^{1}$ - Annalisa Capuano ${ }^{2}$-Salvatore Crisafulli ${ }^{3}$ Paola Maria Cutroneo ${ }^{4}$. Maria Pia Fantini ${ }^{5}$. Carmen Ferrajolo ${ }^{2}$ - Ugo Moretti ${ }^{1}$ - Elisabetta Poluzzi ${ }^{6}$. Emanuel Raschi ${ }^{6} \cdot$ Claudia Ravaldi $^{7}$. Chiara Reno $^{5}$ - Marco Tuccori ${ }^{8}$ - Alfredo Vannacci ${ }^{7}$. Giovanna Zanoni ${ }^{9}$ - Gianluca Trifirò ${ }^{1}$. IImiovaccino COVID19 collaborating group}

1 Section of Pharmacology, Department of Diagnostics and Public Health, University of Verona, Piazzale L.A. Scuro 10, 37134 Verona, Italy

2 Section of Pharmacology “L. Donatelli”, Department of Experimental Medicine, Campania Regional Centre for Pharmacovigilance and Pharmacoepidemiology, University of Campania “Luigi Vanvitelli", Naples, Italy

3 Department of Biomedical and Dental Sciences and Morphofunctional Imaging, University of Messina, Messina, Italy

4 Sicilian Regional Pharmacovigilance Centre, University Hospital of Messina, Messina, Italy
5 Department of Biomedical and Neuromotor Sciences, Alma Mater Studiorum, University of Bologna, Bologna, Italy

6 Department of Medical and Surgical Science, Alma Mater Studiorum, University of Bologna, Bologna, Italy

7 PeaRL-Perinatal Research Laboratory, NEUROFARBA Department, University of Florence and CiaoLapo Foundation for Perinatal Health, Florence, Italy

8 Unit of Adverse Drug Reactions Monitoring, University Hospital of Pisa, Pisa, Italy

9 Immunology Unit, University Hospital, Verona, Italy 